\title{
Analytical Solution for the Constrained Hansen-Jagannathan Distance under Multivariate Ellipticity
}

\author{
Nikolay Gospodinov, Raymond Kan, and Cesare Robotti
}

\author{
Working Paper 2012-18 \\ November 2012
}

\begin{abstract}
We provide an in-depth analysis of the theoretical properties of the Hansen-Jagannathan (HJ) distance that incorporates a no-arbitrage constraint. Under a multivariate elliptical distribution assumption, we present explicit expressions for the HJ-distance with a no-arbitrage constraint, the associated Lagrange multipliers, and the SDF parameters in the case of linear SDFs. This approach allows us to analyze the benefits and costs of using the HJ-distance with a no-arbitrage constraint to rank asset pricing models.
\end{abstract}

JEL classification: G12

Key words: Hansen-Jagannathan distance, no-arbitrage, model ranking, multivariate elliptical distributions

The authors thank Esther Eiling, Wayne Ferson, Jonathan Fletcher, Eric Jondeau, Francisco Penaranda, B. Ravikumar, Sergei Sarkissian, Enrique Sentana, Jonathan Wright, Chu Zhang, Guofu Zhou, seminar participants at the Chinese University of Hong Kong, EDHEC Business School, Emory University, HEC Lausanne, National University of Singapore, Singapore Management University, University of Montreal, University of South Australia, and participants at the 2010 IFM2 Symposium on Mathematical Finance, the 2010 Meetings of the Society for Nonlinear Dynamics and Econometrics, the 2010 International Symposium on Econometric Theory and Applications, and the 2010 Third Annual SoFiE Conference for helpful discussions and comments. Gospodinov gratefully acknowledges financial support from Fonds de recherche sur la société et la culture (FQRSC), Institut de Finance Mathematique de Montreal (IFM2), and the Social Sciences and Humanities Research Council of Canada. Kan gratefully acknowledges financial support from the National Bank Financial of Canada, the Social Sciences and Humanities Research Council of Canada, and the Center for Financial Innovation and Stability at the Federal Reserve Bank of Atlanta. The views expressed here are the authors' and not necessarily those of the Federal Reserve Bank of Atlanta or the Federal Reserve System. Any remaining errors are the authors' responsibility.

Please address questions regarding content to Nikolay Gospodinov, Department of Economics, Concordia University, 1455 de Maisonneuve Boulevard West, Montreal, Quebec H3G 1M8, Canada, and CIREQ, 514-848-2424, 514-848-4536 (fax), nikolay.gospodinov@concordia.ca; Raymond Kan, Joseph L. Rotman School of Management, University of Toronto, 105 St. George Street, Toronto, Ontario M5S 3E6, Canada, kan@chass.utoronto.ca; or Cesare Robotti (contact author), Research Department, Federal Reserve Bank of Atlanta, 1000 Peachtree Street N.E., Atlanta, GA 30309, and EDHEC Risk Institute, cesare.robotti@atl.frb.org.

Federal Reserve Bank of Atlanta working papers, including revised versions, are available on the Atlanta Fed's website at frbatlanta.org/pubs/WP/. Use the WebScriber Service at frbatlanta.org to receive e-mail notifications about new papers. 


\section{Introduction}

Hansen and Jagannathan (1997, HJ hereafter) propose two measures of model misspecification based on the distance between a proposed stochastic discount factor (SDF) and the set of (nonnegative) admissible SDFs (i.e., the set of SDFs that price a given set of test assets correctly). The measure that does not impose the nonnegativity constraint (no-arbitrage condition) on the set of admissible SDFs is referred to as unconstrained HJ-distance whereas the measure that imposes the nonnegativity constraint is referred to as the constrained HJ-distance.

While the unconstrained HJ-distance is analyzed and used in many studies (see, for example, Bansal, Hsieh and Viswanathan (1993), Hansen, Heaton and Luttmer (1995), Campbell and Cochrane (2000), and Kan and Robotti (2009), among others), the constrained HJ-distance has been largely ignored in the literature. Part of the difficulties with the use of the constrained HJdistance is that there is a lack of understanding of the theoretical properties of the constrained HJ-distance due to the fact that an explicit expression for the constrained HJ-distance is not currently available even for linear models.

The main objective of our paper is to provide the readers with a better understanding of the merits and drawbacks of the constrained HJ-distance and the difference between this measure and its unconstrained counterpart. In particular, we derive an explicit solution for the constrained HJ-distance under the assumption that the SDF and the returns are conditionally multivariate elliptically distributed. To facilitate the analysis, we decompose the SDF into a component which is spanned by the returns on the test assets and an unspanned component. This allows us to show that nontrivial differences between the unconstrained and constrained HJ-distances can only arise when the volatility of the unspanned component of an SDF is large and the Sharpe ratio of the tangency portfolio of the test assets is very high. In addition, in the case of linear SDFs, we obtain analytical expressions for the SDF parameters that minimize the constrained HJ-distance. When there is an unspanned factor in the linear SDF, we show that choosing parameters to minimize the constrained HJ-distance instead of the unconstrained HJ-distance results in a lower probability for 
the linear SDF to take on negative values, but leads to a serious deterioration in the ability of the SDF to price the test assets.

The rest of the paper is organized as follows. Section 2 introduces the main setup and notation for the unconstrained and constrained HJ-distances. Section 3 derives an analytical solution for the constrained HJ-distance under the joint ellipticity assumption on the SDF and the returns on the test assets. Some concluding remarks are provided in Section 4.

\section{Unconstrained and Constrained HJ-Distances}

\subsection{Setup and Notation}

Following HJ, let $\mathcal{F}$ be the information that is observed at the date of the asset payoffs. Associated with $\mathcal{F}$ is the space $L^{2}$ of all random variables with finite second moments that are in the information set $\mathcal{F}$. This space is used as the collection of hypothetical claims that could be traded. However, for practical reasons, econometricians can only evaluate asset pricing models on a subspace of $L^{2}$. Let $\tilde{r}=\left[R_{0}, r^{\prime}\right]^{\prime}$, where $R_{0}$ is the gross return on the risk-free asset, and $r$ is a vector of excess returns (in excess of the risk-free rate) on $N$ risky assets. ${ }^{1}$ We assume that the payoff space used in an econometric analysis is given by the payoffs of portfolios of $\tilde{r}$ :

$$
\mathcal{P} \equiv\left\{\omega^{\prime} \tilde{r}: \omega \in \Re^{n}\right\}
$$

where $n=N+1$. In addition, we assume that $E\left[\tilde{r} \tilde{r}^{\prime}\right]$ is nonsingular so that none of the test assets is redundant.

We call $m \in L^{2}$ an admissible SDF if it prices the test assets correctly, i.e.,

$$
E[\tilde{r} m]=q,
$$

where $q=\left[1,0_{N}^{\prime}\right]^{\prime}$ and $0_{N}$ is an $N$-vector of zeros. Let $\mathcal{M}$ denote the set of all admissible SDFs. Although all SDFs in $\mathcal{M}$ can price the test assets correctly, some of them can take on negative

\footnotetext{
${ }^{1}$ It can be readily shown that both the unconstrained and constrained HJ-distances and their SDF parameters are invariant to nonsingular transformations of the return data. Therefore, our results are the same regardless of whether we use excess returns or gross returns on the risky assets. For the case with no risk-free asset, the analysis is slightly more complicated and is available upon request.
} 
values with positive probability and are not consistent with the absence of arbitrage opportunities on the space of hypothetical derivative claims. To eliminate these SDFs from consideration, HJ consider $\mathcal{M}^{+}$, which is the set of nonnegative admissible SDFs.

Let $y \in L^{2}$ be a candidate stochastic discount factor. If $y$ prices the $n$ test assets correctly, then the vector of pricing errors, $e$, of the test assets is exactly zero:

$$
e=E[\tilde{r} y]-q=0_{n}
$$

However, the pricing errors are nonzero when the asset pricing model is misspecified. In this case, we are interested in measuring the degree of model misspecification. HJ suggest using

$$
\delta=\min _{m \in \mathcal{M}}\left(E\left[(y-m)^{2}\right]\right)^{\frac{1}{2}}
$$

as a misspecification measure of $y$. In this paper, we refer to $\delta$ as the unconstrained HJ-distance.

It is possible for an SDF to price all the test assets correctly and yet to take on negative values with positive probability. Such an SDF does not necessarily rule out arbitrage opportunities and it could be problematic to use this SDF to price derivatives on the test assets, for example. To deal with this issue, HJ provide a second model misspecification measure:

$$
\delta_{+}=\min _{m \in \mathcal{M}^{+}}\left(E\left[(y-m)^{2}\right]\right)^{\frac{1}{2}}
$$

We refer to $\delta_{+}$as the constrained HJ-distance. Since $\mathcal{M}^{+}$is a subset of $\mathcal{M}, \delta_{+}$cannot be smaller than $\delta$.

Instead of solving the above primal problems to obtain $\delta$ and $\delta_{+}$, HJ suggest that it is sometimes more convenient to solve the following dual problems:

$$
\begin{aligned}
\delta^{2} & =\max _{\lambda \in \Re^{n}} E\left[y^{2}-\left(y-\lambda^{\prime} \tilde{r}\right)^{2}\right]-2 \lambda^{\prime} q, \\
\delta_{+}^{2} & =\max _{\lambda \in \Re^{n}} E\left[y^{2}-\left[\left(y-\lambda^{\prime} \tilde{r}\right)^{+}\right]^{2}\right]-2 \lambda^{\prime} q,
\end{aligned}
$$

where $\lambda$ is a vector of Lagrange multipliers and $(a)^{+} \equiv \max [a, 0]$. 
When the candidate SDF $y$ depends on some unknown parameters $\gamma$, it is customary to choose $\gamma$ to minimize $\delta$ or $\delta_{+}$, and the squared unconstrained and constrained HJ-distances are then defined as

$$
\begin{aligned}
\delta^{2} & =\min _{\gamma \in \Gamma} \min _{m \in \mathcal{M}} E\left[(y(\gamma)-m)^{2}\right]=\min _{\gamma \in \Gamma} \max _{\lambda \in \Re^{n}} E\left[y(\gamma)^{2}-\left(y(\gamma)-\lambda^{\prime} \tilde{r}\right)^{2}\right]-2 \lambda^{\prime} q, \\
\delta_{+}^{2} & =\min _{\gamma \in \Gamma} \min _{m \in \mathcal{M}^{+}} E\left[(y(\gamma)-m)^{2}\right]=\min _{\gamma \in \Gamma} \max _{\lambda \in \Re^{n}} E\left[y(\gamma)^{2}-\left[\left(y(\gamma)-\lambda^{\prime} \tilde{r}\right)^{+}\right]^{2}\right]-2 \lambda^{\prime} q,
\end{aligned}
$$

where $\Gamma$ is the parameter space of $\gamma$.

\subsection{Analytical Solution for the Unconstrained HJ-Distance}

In this section, we present the explicit expressions for the unconstrained HJ-distance and its associated quantitites. We start with the case where the SDF $y$ does not depend on unknown parameters. The candidate SDF $y$ can always be decomposed into two components:

$$
y=y^{*}+z
$$

where $y^{*}$ is the part of $y$ that is spanned by the returns on the test assets and is given by

$$
y^{*}=\mu_{y}+V_{r y}^{\prime} V_{r r}^{-1}\left(r-\mu_{r}\right)
$$

with $\mu_{y}=E[y], \mu_{r}=E[r], V_{r r}=\operatorname{Var}[r]$, and $V_{r y}=\operatorname{Cov}[r, y]$. It is easy to see that $z$, the unspanned component, has mean zero and is uncorrelated with $r$.

For the unconstrained HJ-distance, the vector of pricing errors of $\tilde{r}$ is given by

$$
e=E[\tilde{r} y]-q=\left[\begin{array}{c}
R_{0} \mu_{y}-1 \\
V_{r y}+\mu_{r} \mu_{y}
\end{array}\right] .
$$

Using the partitioned matrix inverse formula, it is easy to rewrite the inverse of $U=E\left[\tilde{r} \tilde{r}^{\prime}\right]$ as

$$
U^{-1}=\left[\begin{array}{cc}
R_{0}^{2} & R_{0} \mu_{r}^{\prime} \\
R_{0} \mu_{r} & V_{r r}+\mu_{r} \mu_{r}^{\prime}
\end{array}\right]^{-1}=\left[\begin{array}{cc}
\frac{1+a}{R_{0}^{2}} & -\frac{\mu_{r}^{\prime} V_{r r}^{-1}}{R_{0}} \\
-\frac{V_{r r}^{-1} \mu_{r}}{R_{0}} & V_{r r}^{-1}
\end{array}\right],
$$

where $a=\mu_{r}^{\prime} V_{r r}^{-1} \mu_{r}$ is the squared Sharpe ratio of the tangency portfolio of the $N$ risky assets. It follows that the vector of Lagrange multipliers for the unconstrained HJ-distance problem is given 
by

$$
\lambda=U^{-1} e=\left[\begin{array}{c}
\frac{\mu_{y}-V_{r y}^{\prime} V_{r r}^{-1} \mu_{r}}{R_{0}}-\frac{1+a}{R_{0}^{2}} \\
V_{r r}^{-1}\left(V_{r y}+\frac{\mu_{r}}{R_{0}}\right)
\end{array}\right]
$$

and the admissible SDF that is closest to $y$ is

$$
m_{y}=y-\lambda^{\prime} \tilde{r}=z+\frac{1}{R_{0}}-\frac{\mu_{r}^{\prime} V_{r r}^{-1}\left(r-\mu_{r}\right)}{R_{0}} .
$$

After simplification, the squared unconstrained HJ-distance of $y$ is

$$
\delta^{2}=E\left[\left(y-m_{y}\right)^{2}\right]=\left(\mu_{y}-\frac{1}{R_{0}}\right)^{2}+\left(V_{r y}+\frac{\mu_{r}}{R_{0}}\right)^{\prime} V_{r r}^{-1}\left(V_{r y}+\frac{\mu_{r}}{R_{0}}\right) .
$$

When the SDF depends on parameters, we need to solve the outer optimization problem in (8). For general nonlinear SDFs, it is hard to obtain explicit solutions for the SDF parameters, even for the unconstrained HJ-distance. Therefore, we focus on linear SDFs of the form

$$
y(\gamma)=\gamma_{0}+\gamma_{1}^{\prime} f
$$

where $f$ is a vector of $K$ systematic factors, and $\gamma=\left[\gamma_{0}, \gamma_{1}^{\prime}\right]^{\prime}$ is the vector of SDF parameters. In addition to facilitating the derivation of $\gamma$, linear SDFs deserve a thorough investigation because of their popularity in the literature.

Defining $\mu_{f}=E[f]$ and $V_{r f}=\operatorname{Cov}\left[r, f^{\prime}\right]$, it can be readily shown that the parameter vector $\gamma=\left[\gamma_{0}, \gamma_{1}^{\prime}\right]^{\prime}$ that minimizes the unconstrained HJ-distance is

$$
\gamma_{1}=-\frac{1}{R_{0}}\left(V_{r f}^{\prime} V_{r r}^{-1} V_{r f}\right)^{-1}\left(V_{r f}^{\prime} V_{r r}^{-1} \mu_{r}\right), \quad \gamma_{0}=\frac{1}{R_{0}}-\gamma_{1}^{\prime} \mu_{f}
$$

As a result, the linear SDF that minimizes the unconstrained HJ-distance is

$$
y=\frac{1}{R_{0}}+\gamma_{1}^{\prime}\left(f-\mu_{f}\right) .
$$

In addition, defining $a_{1}=\mu_{r}^{\prime} V_{r r}^{-1} V_{r f}\left(V_{r f}^{\prime} V_{r r}^{-1} V_{r f}\right)^{-1} V_{r f}^{\prime} V_{r r}^{-1} \mu_{r}$ as the squared Sharpe ratio of the tangency portfolio constructed from the $K$ factor mimicking portfolios, the squared unconstrained HJ-distance for a linear SDF and the vector of Lagrange multipliers are given by

$$
\delta^{2}=\frac{a-a_{1}}{R_{0}^{2}}
$$


and

$$
\lambda=\left[\begin{array}{c}
-\delta^{2} \\
V_{r r}^{-1}\left(V_{r f} \gamma_{1}+\frac{\mu_{r}}{R_{0}}\right)
\end{array}\right]
$$

\section{Analytical Solution for the Constrained HJ-Distance}

Unlike the case of the unconstrained HJ-distance, obtaining an analytical solution for $\delta_{+}$is infeasible without making a joint distributional assumption on the SDF and the returns on the test assets. More specifically, we assume that the conditional joint distribution of the SDF and the returns is multivariate elliptical (which includes normal, Student $t$, Cauchy, Laplace, symmetric stable, and logistic distributions, among others, as special cases). ${ }^{2}$ It is important to emphasize that while we assume that the conditional joint distribution of the SDF and the returns is multivariate elliptical, we do not make any assumption on their time series properties. The mean and the covariance matrix of the SDF and the returns can be time varying, and many popular time series models like multivariate GARCH with multivariate normal or Student $t$ errors are allowed under our framework. Nevertheless, we do not argue that the multivariate elliptical distribution assumption is always a good approximation of the true conditional distribution of the SDF and the returns. Whether ellipticity provides a reasonable approximation or not depends on the problem at hand. Appendix A provides definitions and notation for elliptically distributed random variables, including some new results on the moments of censored and truncated elliptically distributed random variables. These results are of independent interest given the importance of elliptical distributions for portfolio choice theory, asset and option pricing theory (see Owen and Rabinovitch (1983), Zhou (1993) and Hamada and Valdez (2008), among others.)

\footnotetext{
${ }^{2}$ Since an elliptically distributed SDF takes on negative values by construction, it cannot belong to $\mathcal{M}^{+}$and our theoretical analysis of the constrained HJ-distance is clearly conducted under the hypothesis that the asset pricing model is misspecified.
} 


\subsection{Stochastic discount factors without parameters}

Turning to the constrained HJ-distance case, the vector of Lagrange multipliers in (7) is given by

$$
\tilde{\lambda}=\operatorname{argmin}_{\lambda} E\left[\left(y-\lambda^{\prime} \tilde{r}\right)^{+2}\right]+2 \lambda^{\prime} q
$$

and $\tilde{\lambda}$ can be obtained by solving the following first order condition:

$$
E\left[\tilde{r}\left(y-\tilde{\lambda}^{\prime} \tilde{r}\right)^{+}\right]=q
$$

In principle, we can solve the $n$ nonlinear equations $E\left[\tilde{r}\left(y-\tilde{\lambda}^{\prime} \tilde{r}\right)^{+}\right]=q$ to obtain the vector of Lagrange multipliers $\tilde{\lambda}$, but this can be very complicated. Instead, we simplify the problem so that we only need to solve one nonlinear equation to obtain $\tilde{\lambda}$.

The solution to the first order condition in (23) depends on the joint distribution of $y$ and $r$. Assuming that conditional on $\mathcal{F}, y$ and $r$ have a multivariate elliptical distribution with finite variance, a linear combination of $y$ and $\tilde{r}$, say $v$, also has a conditional elliptical distribution in the same class. We assume that the characteristic function of $v$ can be expressed as $\varphi(t)=$ $\exp \left(i t \mu_{v}\right) \psi\left(t^{2} s_{v}^{2} / 2\right)$ for some function $\psi(\cdot)$, where $\mu_{v}$ is the mean of $v$ and $c^{2} s_{v}^{2}$ is the variance of $v$, with $c=\sqrt{-\psi^{\prime}(0)}$.

We denote the density and cumulative distribution functions of $\tilde{v}=\left(v-\mu_{v}\right) / s_{v}$ as $f$ and $F$, respectively. For a given choice of $f$, we define another elliptically distributed random variable $w$ with the following density function

$$
\tilde{f}(w)=\int_{w}^{\infty} c s f(c s) \mathrm{d} s .
$$

and denote the cumulative distribution function of $w$ by $\tilde{F}$. In Appendix A.1, we present a more complete discussion of the class of elliptical distributions and explicitly derive $\tilde{f}$.

Under the multivariate elliptical distribution assumption, the following proposition presents explicit expressions for the Lagrange multipliers and the squared constrained HJ-distance.

Proposition 1. Suppose $y$ and $r$ are jointly elliptically distributed with finite variance, and $c, F$, 
$\tilde{f}$ and $\tilde{F}$ are defined as above. Let $\eta$ be the unique solution to the following equation

$$
g(u)=\left[a+\sigma_{z}^{2} R_{0}^{2} \tilde{F}(u)^{2}\right]^{-\frac{1}{2}},
$$

where $a$ is the squared Sharpe ratio of the tangency portfolio of the $N$ risky assets, $\sigma_{z}^{2}$ is the variance of the unspanned component of $y$, and

$$
g(u)=\frac{u F(c u)+\tilde{f}(u)}{\tilde{F}(u)} .
$$

The vector of Lagrange multipliers in the constrained HJ-distance case is given by

$$
\tilde{\lambda}=\left[\begin{array}{c}
\frac{\mu_{y}-V_{r y}^{\prime} V_{r r}^{-1} \mu_{r}}{R_{0}}-\frac{a+\frac{\eta}{g(\eta)}}{R_{0}^{2} \tilde{F}(\eta)} \\
V_{r r}^{-1}\left(V_{r y}+\frac{\mu_{r}}{R_{0} \tilde{F}(\eta)}\right)
\end{array}\right] .
$$

The squared constrained HJ-distance of an SDF y is given by

$$
\delta_{+}^{2}=\delta^{2}+\sigma_{z}^{2} \tilde{F}(-\eta)+\frac{a+\frac{\eta}{g(\eta)}}{R_{0}^{2} \tilde{F}(\eta)}-\frac{1+a}{R_{0}^{2}},
$$

where the expression of $\delta^{2}$ is provided in (16).

Proof. See Appendix B.

The $\mathrm{SDF}$ in $\mathcal{M}^{+}$that is closest to $y$ is $m_{y}^{+}$, where

$$
m_{y}=y-\tilde{\lambda}^{\prime} \tilde{r}=z+\frac{1}{R_{0} \tilde{F}(\eta)}\left[\frac{\eta}{g(\eta)}-\mu_{r}^{\prime} V_{r r}^{-1}\left(r-\mu_{r}\right)\right]
$$

Just like $m_{y}$ in (15) for the unconstrained HJ-distance case, the $m_{y}$ for the constrained HJ-distance has two components. The first component, $z$, is uncorrelated with the returns, and the second component is a linear function of the excess return on the tangency portfolio of the test assets. Let $\Delta=\delta_{+}^{2}-\delta^{2}$ be the difference between the squared constrained and unconstrained HJ-distances of $y$. We are interested in the determinants of $\Delta$. Note that $\eta$, the solution to (25), depends on $\sigma_{z}^{2}$, $a$, and $R_{0}$. As a result, $\Delta$ also depends on these three parameters. Out of the three parameters, the only one that is related to the $\mathrm{SDF}$ is $\sigma_{z}^{2}$ - the variance of the unspanned component. When $\sigma_{z}^{2}=0$ (i.e., spanned SDF), we have

$$
g(\eta)=\frac{1}{\sqrt{a}}
$$


and hence

$$
\Delta=\frac{a+\sqrt{a} \eta}{R_{0}^{2} \tilde{F}(\eta)}-\frac{1+a}{R_{0}^{2}}=\left[\frac{\sqrt{a}(\sqrt{a}+\eta)}{R_{0}^{2} \tilde{F}(\eta)}-\frac{1}{R_{0}^{2}}\right]-\frac{a}{R_{0}^{2}}=\sigma_{c}^{2}-\sigma_{0}^{2},
$$

where $\sigma_{c}^{2}=\min _{m \in \mathcal{M}^{+}} \operatorname{Var}[m]$ and $\sigma_{0}^{2}=\min _{m \in \mathcal{M}} \operatorname{Var}[m]$ are the constrained and unconstrained bounds of Hansen and Jagannathan (1991). ${ }^{3}$ It should be noted that both HJ-bounds (and their difference) only depend on the choice of the test assets and are model independent. Therefore, when the SDF is spanned, $\delta_{+}^{2}-\delta^{2}$ is also model independent. This result implies that for two spanned SDFs, the difference between their unconstrained HJ-distances is the same as the difference between their constrained HJ-distances. Therefore, for spanned SDFs, the constrained and unconstrained HJ-distances will provide the same ranking of competing asset pricing models.

The following lemma provides the comparative statics of $\Delta$ with respect to its three determinants.

Lemma 1. The partial derivatives of $\Delta$ with respect to $\left(\sigma_{z}^{2}, a, R_{0}\right)$ are given by

$$
\begin{aligned}
\frac{\partial \Delta}{\partial \sigma_{z}^{2}} & =\tilde{F}(-\eta)>0 \\
\frac{\partial \Delta}{\partial a} & =\frac{\tilde{F}(-\eta)}{R_{0}^{2} \tilde{F}(\eta)}>0 \\
\frac{\partial \Delta}{\partial R_{0}} & =\frac{2}{R_{0}^{3}}\left[1-\frac{a \tilde{F}(-\eta)}{\tilde{F}(\eta)}-\frac{\eta}{\tilde{F}(\eta) g(\eta)}\right] .
\end{aligned}
$$

Proof. See Appendix B.

Lemma 1 shows that $\Delta$ is an increasing function of $\sigma_{z}^{2}$, which suggests that $\sigma_{c}^{2}-\sigma_{0}^{2}$ is a lower bound for $\Delta$. Intuitively, adding an unspanned component $z$ to an SDF does not affect its ability to price the test assets, so the unconstrained HJ-distance (which is a measure of aggregate pricing errors) of a model is unaffected by $z$. As a result, the expression of $\delta^{2}$ in (16) is independent of $\sigma_{z}^{2}$. However, adding an elliptically distributed $z$ to an SDF can affect the SDF's probability of taking on negative values and hence $\Delta$ is an increasing function of $\sigma_{z}^{2}$. Note that $\Delta$ does not depend on

\footnotetext{
${ }^{3}$ The last equality in (31) is a general result. It is not specific to the ellipticity assumption on the SDF and the returns or to assuming, as we do in this section, that the SDF does not depend on parameters (more on this in Section 3.2).
} 
how good or bad a model is for the test assets. It is only a function of the variance of its unspanned component. This suggests that for two different models, say $\mathcal{F}$ and $\mathcal{G}$, we can expect $\delta_{\mathcal{F},+}^{2}-\delta_{\mathcal{G},+}^{2}$ to differ substantially from $\delta_{\mathcal{F}}^{2}-\delta_{\mathcal{G}}^{2}$ only when the variances of the unspanned components across the two models are very different.

Furthermore, Lemma 1 suggests that $\Delta$ is an increasing function of the Sharpe ratio of the tangency portfolio of the test assets. This result requires some explanation. Consider the case in which $\sigma_{z}^{2} \rightarrow 0$. When this happens, $\left(\delta_{+}^{2}-\delta^{2}\right) \rightarrow\left(\sigma_{c}^{2}-\sigma_{0}^{2}\right)$ - the difference between the constrained and unconstrained HJ-bounds. Lemma 6 of Kan and Robotti (2008) shows that, under normality, $\left(\sigma_{c}^{2}-\sigma_{0}^{2}\right) \rightarrow 0$ when $a \rightarrow 0,\left(\sigma_{c}^{2}-\sigma_{0}^{2}\right) \rightarrow \infty$ when $a \rightarrow \infty$, and $\sigma_{c}^{2}-\sigma_{0}^{2}$ is a strictly increasing function of $a$. Therefore, when $a$ is small, we should not expect large differences between the constrained and unconstrained HJ-bounds and between the constrained and unconstrained HJ-distances. Intuitively, when $a$ is close to zero, the weight of the risk-free asset in the minimum second moment portfolio is close to one, and the gross return on this portfolio has a very small probability of taking on a negative value. Since the minimum variance admissible SDF is proportional to the gross return on this portfolio, imposing the nonnegativity constraint of Hansen and Jagannathan (1991) on it has almost no effect.

\section{Figure 1 about here}

To gain some understanding of how $\sigma_{z}$ and $a$ affect $\Delta$, Figure 1 plots $\Delta$ as a function of $\sigma_{z}$ for three different values of the Sharpe ratio of the tangency portfolio $(\sqrt{a}=0.25,0.5$, and 0.75$)$ with $R_{0}=1.005$ (the plot is not sensitive to other reasonable values of the gross risk-free rate). In the plot, the SDF and the excess returns on the test assets are assumed to be multivariate $t$-distributed with six degrees of freedom. As expected, Figure 1 reveals that $\Delta$ is an increasing function of $\sigma_{z}$. However, $\Delta$ is heavily influenced by the Sharpe ratio of the tangency portfolio. When $\sqrt{a}=0.25$, the difference between $\delta_{+}^{2}$ and $\delta^{2}$ is indistinguishable from zero. For $\sqrt{a}=0.5$, the difference between $\delta_{+}^{2}$ and $\delta^{2}$ is still quite small, even for relatively large $\sigma_{z}$. This suggests that for reasonable 
Sharpe ratio values, we should not expect to find a large difference between the constrained and unconstrained HJ-distances of a model, even if the model contains a large unspanned component.

\subsection{Linear stochastic discount factors}

Consider the linear SDF (17). We first define the covariance matrix of the residuals from projecting the factors onto the returns as $V_{f f \cdot r}=V_{f f}-V_{r f}^{\prime} V_{r r}^{-1} V_{r f}$, where $V_{f f}=\operatorname{Var}[f]$. The following proposition presents the solution to the constrained HJ-distance problem.

Proposition 2. Let $\eta$ be the unique solution to

$$
g(u)=\left(a+\alpha^{\prime}\left[\frac{1}{\tilde{F}(u)} I_{K}-V_{f f}^{-\frac{1}{2}} V_{f f \cdot r} V_{f f}^{-\frac{1}{2}}\right]^{-2} \alpha\right)^{-\frac{1}{2}}
$$

where $\alpha=V_{f f}^{-1} V_{f f \cdot r}^{\frac{1}{2}} V_{r f}^{\prime} V_{r r}^{-1} \mu_{r}, g(u)$ is defined in (26), and $\tilde{F}$ is defined before Proposition 1. Then, the vector of SDF parameters that minimizes the constrained HJ-distance is given by $\tilde{\gamma}=\left[\tilde{\gamma}_{0}, \tilde{\gamma}_{1}^{\prime}\right]^{\prime}$, where

$$
\tilde{\gamma}_{1}=-\frac{1}{R_{0}}\left[V_{f f}-\tilde{F}(\eta) V_{f f \cdot r}\right]^{-1}\left(V_{r f}^{\prime} V_{r r}^{-1} \mu_{r}\right), \quad \tilde{\gamma}_{0}=\frac{1}{R_{0}}-\tilde{\gamma}_{1}^{\prime} \mu_{f}
$$

and the SDF that minimizes the constrained HJ-distance is

$$
\tilde{y}=\frac{1}{R_{0}}+\tilde{\gamma}_{1}^{\prime}\left(f-\mu_{f}\right)
$$

Furthermore, the squared constrained HJ-distance has the following expression:

$$
\delta_{+}^{2}=\frac{a+\frac{\eta}{g(\eta)}}{R_{0}^{2} \tilde{F}(\eta)}-\frac{1+\tilde{a}_{1}}{R_{0}^{2}}
$$

where $\tilde{a}_{1}=\mu_{r}^{\prime} V_{r r}^{-1} V_{r f}\left[V_{f f}-\tilde{F}(\eta) V_{f f \cdot r}\right]^{-1} V_{r f}^{\prime} V_{r r}^{-1} \mu_{r}$ and the vector of Lagrange multipliers for the constrained HJ-distance is given by

$$
\tilde{\lambda}=\left[\begin{array}{c}
-\delta_{+}^{2} \\
V_{r r}^{-1}\left(V_{r f} \tilde{\gamma}_{1}+\frac{\mu_{r}}{R_{0} \tilde{F}(\eta)}\right)
\end{array}\right]
$$

Proof. See Appendix B. 
Besides the simplicity of the expressions for $\tilde{\gamma}, \tilde{\lambda}$, and $\delta_{+}^{2}$, a few interesting observations emerge from Proposition 2. First, the Lagrange multiplier on the risk-free asset is equal to $-\delta_{+}^{2}$ (expression (21) shows that a similar result holds for the unconstrained HJ-distance). Second, in contrast to the SDF case without parameters, $\eta$ does not depend on $R_{0}$ since $\tilde{y}$ prices the risk-free asset correctly. Third, when the factors are spanned by the returns (i.e., $V_{f f \cdot r}=0_{K \times K}$ ), it can be readily shown that the difference between the squared constrained and unconstrained HJ-distances coincides with the difference between the constrained and unconstrained HJ-bounds. This result confirms our earlier findings for spanned SDFs in Section 3.1. Finally, when one or more factors are useless, i.e., they are uncorrelated with the returns, the SDF parameters that minimize the unconstrained HJ-distance are not identified since the matrix $V_{r f}^{\prime} V_{r r}^{-1} V_{r f}$ is not of full rank and cannot be inverted. However, the SDF parameters that minimize the constrained HJ-distance are still well defined. For example, when all factors are useless, we have $\tilde{\gamma}=\left[1 / R_{0}, 0_{K}^{\prime}\right]^{\prime}$ and $\tilde{y}=1 / R_{0}$. In this case, $\delta_{+}^{2}$ is equal to the constrained HJ-bound $\sigma_{c}^{2}$.

With the analytical solutions of the linear SDF parameters for the unconstrained and constrained HJ-distances, we can now answer two interesting questions. The first question is whether the linear $\mathrm{SDF} \tilde{y}$ in (37) results in a lower probability of taking on negative values than the linear SDF $y$ in (19). If this is the case, one can think of this as a potential benefit of using the constrained HJ-distance. The second question is whether there is a trade-off between getting the linear SDF closer to $\mathcal{M}^{+}$and the ability of the SDF to price the test assets. For this purpose, we introduce an aggregate measure of pricing errors of $\tilde{y}$ as

$$
\tilde{\delta}^{2}=\tilde{e}^{\prime} U^{-1} \tilde{e}
$$

where $\tilde{e}=E[\tilde{r} \tilde{y}]-q$ is the vector of pricing errors when we use $\tilde{y}$ to price the test assets. Just like the $\delta^{2}$ measure, $\tilde{\delta}^{2}$ can be interpreted as the maximum squared pricing error of a portfolio of test assets when one uses $\tilde{y}$ as the SDF. Comparing $\tilde{\delta}^{2}$ with $\delta^{2}$, we gain useful insights of the potential cost of using $\tilde{y}$ instead of $y$ to price the test assets. The following lemma provides answers to these two questions. 
Lemma 2. Let $y$ and $\tilde{y}$ be the linear SDFs that minimize the unconstrained and constrained HJ-distances, respectively. Then, we have

$$
P[y<0]-P[\tilde{y}<0]=F\left(-\frac{c}{R_{0} \sqrt{\gamma_{1}^{\prime} V_{f f} \gamma_{1}}}\right)-F\left(-\frac{c}{R_{0} \sqrt{\tilde{\gamma}_{1}^{\prime} V_{f f} \tilde{\gamma}_{1}}}\right)>0,
$$

where $F$ and $c$ are defined before Proposition 1. In addition, we have

$$
\delta^{2} \leq \tilde{\delta}^{2} \leq \delta_{+}^{2}
$$

Proof. See Appendix B.

As shown in the proof of Lemma 2, (42) is a general result. It is not specific to the linear model and our proof does not rely on the ellipticity assumption. However, we can only establish $P[y<$ $0]>P[\tilde{y}<0]$ for the case of linear models and under the ellipticity assumption.

Lemma 2 suggests that there are potential benefits and costs in choosing the SDF parameters to minimize the constrained HJ-distance as opposed to minimizing the unconstrained HJ-distance. On the one hand, $\tilde{y}$ is less likely than $y$ to take on negative values. On the other hand, $\tilde{y}$ will price the test assets worse than $y$. Exactly how large is this cost-benefit trade-off depends on the parameters. For the one-factor case, we can show that

$$
P[y<0]-P[\tilde{y}<0]=F\left(-\frac{c|\rho|}{\sqrt{a_{1}}}\right)-F\left(-\frac{c\left[1-\tilde{F}(\eta)\left(1-\rho^{2}\right)\right]}{\sqrt{a_{1}}|\rho|}\right),
$$

where $\eta$ is the unique solution to

$$
g(u)=\left[a+\frac{a_{1} \rho^{2}\left(1-\rho^{2}\right) \tilde{F}(u)^{2}}{\left[1-\tilde{F}(u)\left(1-\rho^{2}\right)\right]^{2}}\right]^{-\frac{1}{2}}
$$

and $\rho^{2}=V_{r f}^{\prime} V_{r r}^{-1} V_{r f} / V_{f f}$ is the proportion of variability of the factor that is explained by the returns on the test assets. In addition, we have

$$
\tilde{\delta}^{2}-\delta^{2}=\frac{a_{1}}{R_{0}^{2}}\left[\frac{\tilde{F}(-\eta)\left(1-\rho^{2}\right)}{1-\tilde{F}(\eta)\left(1-\rho^{2}\right)}\right]^{2}
$$


Note that both (43) and (45) depend on $a, a_{1}$ and $\rho^{2}$. In these expressions, $a$ is the squared Sharpe ratio of the tangency portfolio of the test assets, which is a measure of the cross-sectional difference in expected excess returns across the test assets; $a_{1}$ measures how good the model is in explaining the expected returns on the test assets (recall that $\left.\delta^{2}=\left(a-a_{1}\right) / R_{0}^{2}\right)$; and, finally, $\rho^{2}$ measures how well the factor is spanned by the returns.

Assuming that the factor and the excess returns on the test are multivariate $t$-distributed with six degrees of freedom, Figure 2 plots $P[y<0]-P[\tilde{y}<0]$ as a function of $\rho^{2}$ for three different values of the Sharpe ratio of the tangency portfolio $(\sqrt{a}=0.25,0.5$, and 0.75$)$. In each case, we assume $a_{1}=a / 2$, so that the model explains half of the cross-sectional variation in expected returns.

\section{Figure 2 about here}

From Figure 2, we can see that when $\rho^{2} \rightarrow 0$ ( $y$ is not defined when $\left.\rho^{2}=0\right), P[y<0]-P[\tilde{y}<$ $0] \rightarrow 0.5$. The reason is that when the unspanned component of the factor increases, $y$ becomes more volatile (because $\gamma_{1}$ does not depend on the unspanned component of the factor) and behaves more like a useless factor. As a result, $P[y<0] \rightarrow 0.5$. However, as $\rho^{2} \rightarrow 0, \tilde{y}$ converges to $1 / R_{0}$ and has almost zero probability of taking on negative values. In contrast, when $\rho^{2} \rightarrow 1$, the SDF behaves more like a spanned SDF. For a spanned SDF, the SDF parameters and hence the probabilities of taking on negative values are the same for $y$ and $\tilde{y}$. Finally, Figure 3 shows that the Sharpe ratio is important in determining $P[y<0]-P[\tilde{y}<0]$. For a given value of $\rho^{2}$, we can see that the difference between the two probabilities is an increasing function of $a$. The reason is that the spanned component of the SDF $y$ is a linear function of the return on the factor mimicking portfolio. When $a$ is small, $a_{1}$ is also small, so $y$ puts relatively little weight on the factor mimicking portfolio and hence $P[y<0]$ is small, leaving not much room for $\tilde{y}$ to improve.

Using the same parameters and distributional assumption as in Figure 2, Figure 3 plots $\tilde{\delta}^{2}-\delta^{2}$ as a function of $\rho^{2}$ for $R_{0}=1.005$. 
Figure 3 about here

Again, when $\rho^{2} \approx 1$, the SDF is close to a spanned one. It follows that $y \approx \tilde{y}$, so they have roughly the same aggregate pricing errors and $\tilde{\delta}^{2}-\delta^{2} \rightarrow 0$. However, when $\rho^{2} \rightarrow 0$, we have $\tilde{\delta}^{2}=a / R_{0}^{2}\left(\right.$ as $\tilde{y} \approx 1 / R_{0}$ and $\tilde{y}$ does not explain any cross-sectional difference in expected excess returns). It follows that $\tilde{\delta}^{2}-\delta^{2} \rightarrow a_{1} / R_{0}^{2}$. Similar to Figure 2 , we also find $a$ to be quite important in determining $\tilde{\delta}^{2}-\delta^{2}$. It is only when $a$ is large (and hence $a_{1}$ is large) that we should expect a large difference between the aggregate measures of pricing errors of $y$ and $\tilde{y}$.

In summary, we should expect $y$ and $\tilde{y}$ to behave differently if $a$ is large and $\rho^{2}$ is small. In these situations, $P[\tilde{y}<0]$ will be substantially smaller than $P[y<0]$, but these are also situations in which $\tilde{y}$ will do substantially worse than $y$ in pricing the test assets. Whether one should sacrifice the pricing of the test assets in exchange for a smaller SDF's probability of taking on negative values is not entirely clear. For example, when $\rho^{2}$ is small, $\tilde{y} \approx 1 / R_{0}$ and $\tilde{y}$ is indeed almost always positive. However, this $\tilde{y}$ is unlikely to be a good SDF even for pricing derivatives since it prices every asset by discounting the future asset payoffs using the risk-free rate.

\section{Conclusion}

In this paper, we derive an analytical solution for the constrained HJ-distance, the associated Lagrange multipliers, and the SDF parameters in the case of linear SDFs under an ellipticity assumption on the conditional joint distribution of the SDF and the returns. This allows us to show that nontrivial differences between the constrained and unconstrained HJ-distances can only arise when the volatility of the unspanned component of an SDF is large and the Sharpe ratio of the tangency portfolio of the test assets is very high. In addition, our analysis allows us to quantify the deterioration in the ability of a given linear SDF to price the test assets when imposing a no-arbitrage constraint. 


\section{Appendix A: Definitions and Preliminary Lemmas}

\section{A.1 Elliptical distributions: Definitions and notation}

In this section, we introduce the definitions and notation for the class of multivariate elliptical distributions, following closely Landsman and Valdez (2003). We say that two random variables $(u, v)$ have a bivariate elliptical distribution, written as $\mathcal{E}(\mu, S, \psi)$, if their characteristic function can be expressed as

$$
\varphi(t)=\exp \left(i t^{\prime} \mu\right) \psi\left(\frac{t^{\prime} S t}{2}\right)
$$

for some

$$
\mu=\left[\begin{array}{l}
\mu_{u} \\
\mu_{v}
\end{array}\right], \quad S=\left[\begin{array}{cc}
s_{u}^{2} & s_{u v} \\
s_{v u} & s_{v}^{2}
\end{array}\right]
$$

and $\psi(\cdot)$, which is called the characteristic generator. When the mean of $[u, v]^{\prime}$ exists, we have $E[u]=\mu_{u}$ and $E[v]=\mu_{v}$. When the variance of $[u, v]^{\prime}$ exists, we have $\sigma_{u}^{2}=\operatorname{Var}[u]=c^{2} s_{u}^{2}$, $\sigma_{v}^{2}=\operatorname{Var}[v]=c^{2} s_{v}^{2}$ and $\sigma_{u v}=\operatorname{Cov}[u, v]=c^{2} s_{u v}$, where $c=\sqrt{-\psi^{\prime}(0)}$. It is important to remember that $S$ is not the covariance matrix of $[u, v]^{\prime}$ in general.

We assume that the density functions of $u$ and $v$ exist. The density function of $v$ (the density function of $u$ is similarly defined) is given by

$$
f_{v}(v)=\frac{c_{1}}{s_{v}} h\left(\frac{\left(v-\mu_{v}\right)^{2}}{2 s_{v}^{2}}\right)
$$

where $h(\cdot)$ is a nonnegative function (called the density generator) and

$$
c_{1}=\frac{1}{\sqrt{2}}\left[\int_{0}^{\infty} x^{-\frac{1}{2}} h(x) \mathrm{d} x\right]^{-1}
$$

is a normalization constant.

We provide two examples of elliptical distributions: normal and Student $t$. For the normal 
distribution, we have

$$
\begin{aligned}
\psi(s) & =e^{-s}, \\
h(t) & =e^{-t}, \\
c_{1} & =\frac{1}{\sqrt{2 \pi}}, \\
f_{v}(v) & =\frac{1}{\sqrt{2 \pi} s_{v}} e^{-\frac{\left(v-\mu_{v}\right)^{2}}{2 s_{v}^{2}}} .
\end{aligned}
$$

It follows that $\psi^{\prime}(s)=-e^{-s}, c=\sqrt{-\psi^{\prime}(0)}=1$, and $\sigma_{v}^{2}=s_{v}^{2}$.

For the Student $t$ distribution with $\nu$ degrees of freedom, we have

$$
\begin{aligned}
\psi(s) & =\frac{\mathrm{K}_{\nu / 2}(\sqrt{2 \nu s})\left(\frac{\nu s}{2}\right)^{\frac{\nu}{4}}}{2 \Gamma\left(\frac{\nu}{2}\right)}, \\
h(t) & =\left(1+\frac{2 t}{\nu}\right)^{-\frac{\nu+1}{2}}, \\
c_{1} & =\frac{1}{\mathrm{~B}\left(\frac{1}{2}, \frac{\nu}{2}\right) \sqrt{\nu}}, \\
f_{v}(v) & =\frac{1}{\mathrm{~B}\left(\frac{1}{2}, \frac{\nu}{2}\right) \sqrt{\nu} s_{v}}\left[1+\frac{\left(v-\mu_{v}\right)^{2}}{\nu s_{v}^{2}}\right]^{-\frac{\nu+1}{2}},
\end{aligned}
$$

where $\mathrm{K}_{\nu}(x)$ is the modified Bessel function of the second kind, $\Gamma(a)$ is the gamma function, and $\mathrm{B}(a, b)$ is the beta function. It is straightforward to show that

$$
\psi^{\prime}(s)=-\frac{\nu \mathrm{K}_{(\nu-2) / 2}(\sqrt{2 \nu s})\left(\frac{\nu s}{2}\right)^{\frac{\nu-2}{4}}}{\Gamma\left(\frac{\nu}{2}\right)},
$$

and $c=\sqrt{-\psi^{\prime}(0)}=[\nu /(\nu-2)]^{\frac{1}{2}}$. In addition, when $\nu>1$, the mean of $v$ exists and when $\nu>2$, the variance of $v$ exists and $\sigma_{v}^{2}=\nu s_{v}^{2} /(\nu-2)$.

For a given elliptical random variable $v$ with parameters $\mu_{v}$ and $s_{v}^{2}$, we define

$$
\tilde{v}=\frac{v-\mu_{v}}{s_{v}}
$$

The random variable $\tilde{v}$ has a spherical distribution (i.e., an elliptical distribution with parameters $\mu_{\tilde{v}}=0$ and $\left.s_{\tilde{v}}=1\right)$. We denote its density and cumulative distribution functions by $f(\tilde{v})$ and $F(\tilde{v})$, respectively. Note that

$$
f(\tilde{v})=c_{1} h\left(\frac{\tilde{v}^{2}}{2}\right) .
$$


By symmetry, we have $f(-\tilde{v})=f(\tilde{v})$ and $1-F(-\tilde{v})=F(\tilde{v})$. In addition, we have $\sigma_{\tilde{v}}^{2}=c^{2}$ when the variance of $\tilde{v}$ exists.

For every spherical random variable $\tilde{v}$ with finite variance, Landsman and Valdez (2003) show that a random variable $w$ with the following density function ${ }^{4}$

$$
\tilde{f}(w)=\int_{w}^{\infty} c \tilde{v} f(c \tilde{v}) \mathrm{d} \tilde{v}=\frac{1}{c} \int_{c w}^{\infty} s f(s) \mathrm{d} s
$$

is also a spherical random variable. The density function of $w$ can alternatively be written as

$$
\tilde{f}(w)=\frac{c_{1}}{c} H\left(\frac{c^{2} w^{2}}{2}\right)
$$

where

$$
H(x)=\int_{x}^{\infty} h(t) \mathrm{d} t
$$

From this expression, we can easily see that the density function of $w$ only depends on $w^{2}$, so $w$ has a spherical distribution. The distribution of $w$ is crucial for us to obtain the tail conditional expectation of $v$.

For a given spherical random variable $\tilde{v}$, the above definitions allow us to quickly obtain the density function of the associated spherical random variable $w$. For example, when $\tilde{v} \sim N(0,1)$, we have $h(t)=e^{-t}$ and

$$
H(x)=\int_{x}^{\infty} e^{-t} \mathrm{~d} t=e^{-x} .
$$

Therefore, using $c=1$ and $c_{1}=1 / \sqrt{2 \pi}$, we obtain

$$
\tilde{f}(w)=\frac{1}{\sqrt{2 \pi}} e^{-\frac{w^{2}}{2}}
$$

and $w \sim N(0,1)$.

When $\tilde{v} \sim t_{\nu}$ for $\nu>2$, we use (A10) to obtain

$$
H(x)=\int_{x}^{\infty}\left(1+\frac{2 t}{\nu}\right)^{-\frac{\nu+1}{2}} \mathrm{~d} t=\frac{\nu}{\nu-1}\left(1+\frac{2 x}{\nu}\right)^{-\frac{\nu-1}{2}} .
$$

\footnotetext{
${ }^{4}$ Instead of mapping $\tilde{v}$ to $w$, Landsman and Valdez (2003) define a slightly different mapping from $\tilde{v}$ to $Z^{*}=c w$.
} 
Then using (A11) and $c=[\nu /(\nu-2)]^{\frac{1}{2}}$, we obtain

$$
\begin{aligned}
\tilde{f}(w) & =\frac{1}{\sqrt{\nu} \mathrm{B}\left(\frac{1}{2}, \frac{\nu}{2}\right)} \frac{\sqrt{\nu-2}}{\sqrt{\nu}} \frac{\nu}{\nu-1}\left(1+\frac{w^{2}}{\nu-2}\right)^{-\frac{\nu-1}{2}} \\
& =\frac{1}{\sqrt{\nu-2} \mathrm{~B}\left(\frac{1}{2}, \frac{\nu-2}{2}\right)}\left(1+\frac{w^{2}}{\nu-2}\right)^{-\frac{\nu-1}{2}},
\end{aligned}
$$

and $w \sim t_{\nu-2}$.

\section{A.2 Preliminary lemmas}

Lemma A.1. Suppose $[u, v]^{\prime}$ is bivariate elliptically distributed with finite variance. Let $\eta=\mu_{v} / \sigma_{v}$, where $\mu_{v}$ and $\sigma_{v}$ are the mean and standard deviation of $v$, respectively. We have

$$
\begin{aligned}
E\left[v^{+}\right] & =\mu_{v} F(c \eta)+\sigma_{v} \tilde{f}(\eta)=\tilde{F}(\eta) \sigma_{v} g(\eta), \\
E\left[u v^{+}\right] & =\sigma_{u v} \tilde{F}(\eta)+\mu_{u}\left[\mu_{v} F(c \eta)+\sigma_{v} \tilde{f}(\eta)\right] \\
& =\tilde{F}(\eta)\left(E[u v]+\mu_{u} \sigma_{v}[g(\eta)-\eta]\right),
\end{aligned}
$$

where $F$ is the cumulative distribution function of $\tilde{v}=\left(v-\mu_{v}\right) / s_{v}, c=\sigma_{\tilde{v}}, \tilde{f}$ and $\tilde{F}$ are the density and cumulative distribution functions of another spherical random variable $w$ that is associated with $\tilde{v}$ as defined in (A16), and

$$
g(\eta)=\frac{\eta F(c \eta)+\tilde{f}(\eta)}{\tilde{F}(\eta)}
$$

Proof of Lemma A.1. For a given $f$, we define the functions

$$
\begin{aligned}
\tilde{h}(x) & =\int_{-x}^{\infty} \tilde{v} f(\tilde{v}) \mathrm{d} \tilde{v} \\
\tilde{H}(x) & =\int_{-x}^{\infty} \tilde{v}^{2} f(\tilde{v}) \mathrm{d} \tilde{v} .
\end{aligned}
$$

We are interested in obtaining $E\left[v^{+}\right]$, which is given by

$$
E\left[v^{+}\right]=\int_{0}^{\infty} v f_{v}(v) \mathrm{d} v=\int_{-c \eta}^{\infty}\left(\mu_{v}+s_{v} \tilde{v}\right) f(\tilde{v}) \mathrm{d} \tilde{v}=\mu_{v} F(c \eta)+s_{v} \tilde{h}(c \eta)=\mu_{v} F(c \eta)+\sigma_{v} \tilde{f}(\eta)
$$

where the last equality follows from (A16) and the fact that $\tilde{h}(c \eta)=c \tilde{f}(-\eta)=c \tilde{f}(\eta)$. 
In order to obtain $E\left[u v^{+}\right]$, we need to first derive $E\left[v^{+2}\right]$, which is given by

$$
E\left[v^{+2}\right]=\int_{0}^{\infty} v^{2} f_{v}(v) \mathrm{d} v=\int_{-c \eta}^{\infty}\left(\mu_{v}+s_{v} \tilde{v}\right)^{2} f(\tilde{v}) \mathrm{d} \tilde{v}=\mu_{v}^{2} F(c \eta)+2 \mu_{v} s_{v} \tilde{h}(c \eta)+s_{v}^{2} \tilde{H}(c \eta) .
$$

Since

$$
\frac{\mathrm{d} \tilde{h}(\eta)}{\mathrm{d} \eta}=\eta f(-\eta)=\eta f(\eta)
$$

we can use integration by parts to obtain

$$
\begin{aligned}
\tilde{H}(c \eta) & =\int_{-c \eta}^{\infty} \tilde{v}^{2} f(\tilde{v}) \mathrm{d} \tilde{v} \\
& =\left.\tilde{v} \tilde{h}(\tilde{v})\right|_{-c \eta} ^{\infty}-\int_{-c \eta}^{\infty} \tilde{h}(\tilde{v}) \mathrm{d} \tilde{v} \\
& =d-c \eta \tilde{h}(c \eta)-c \int_{-c \eta}^{\infty} \tilde{f}\left(\frac{\tilde{v}}{c}\right) \mathrm{d} \tilde{v} \\
& =d-c^{2} \eta \tilde{f}(\eta)-c^{2} \int_{-\eta}^{\infty} \tilde{f}(s) \mathrm{d} s \\
& =d-c^{2} \eta \tilde{f}(\eta)+c^{2} \tilde{F}(\eta),
\end{aligned}
$$

where $d \equiv \lim _{\tilde{v} \rightarrow \infty} \tilde{v} \tilde{h}(\tilde{v})$. We now show that $d=0$ when $c<\infty$. Since $w$ is a symmetric random variable, $\tilde{F}(0)=1 / 2$ and it follows that

$$
\tilde{H}(0)=d+c^{2} \tilde{F}(0)=d+\frac{c^{2}}{2} .
$$

However, we know that

$$
\tilde{H}(0)=\int_{0}^{\infty} \tilde{v}^{2} f(\tilde{v}) \mathrm{d} \tilde{v}=\frac{c^{2}}{2},
$$

and hence $d=0$ when $c$ is finite. Therefore, we have

$$
\tilde{H}(c \eta)=-c^{2} \eta \tilde{f}(c \eta)+c^{2} \tilde{F}(\eta) .
$$

Using (A29) and $\tilde{h}(c \eta)=c \tilde{f}(\eta)$, we have

$$
\begin{aligned}
E\left[v^{+2}\right] & =\mu_{v}^{2} F(c \eta)+2 \mu_{v} s_{v} \tilde{h}(c \eta)-s_{v}^{2} c^{2} \eta \tilde{f}(\eta)+s_{v}^{2} c^{2} \tilde{F}(\eta) \\
& =\mu_{v}^{2} F(c \eta)+\mu_{v} \sigma_{v} \tilde{f}(\eta)+\sigma_{v}^{2} \tilde{F}(\eta)
\end{aligned}
$$


Under the bivariate elliptical assumption on $u$ and $v$, we have

$$
E[u \mid v]=\mu_{u}+\frac{\sigma_{u v}}{\sigma_{v}^{2}}\left(v-\mu_{v}\right)
$$

It then follows that

$$
\begin{aligned}
E\left[u v^{+}\right] & =E\left[E[u \mid v] v^{+}\right] \\
& =E\left[\left(\mu_{u}+\frac{\sigma_{u v}}{\sigma_{v}^{2}}\left(v-\mu_{v}\right)\right) v^{+}\right] \\
& =\left(\mu_{u}-\frac{\sigma_{u v}}{\sigma_{v}^{2}} \mu_{v}\right) E\left[v^{+}\right]+\frac{\sigma_{u v}}{\sigma_{v}^{2}} E\left[v^{+2}\right] \\
& =\left(\mu_{u}-\frac{\sigma_{u v}}{\sigma_{v}^{2}} \mu_{v}\right)\left[\mu_{v} F(c \eta)+\sigma_{v} \tilde{f}(\eta)\right]+\frac{\sigma_{u v}}{\sigma_{v}^{2}}\left[\mu_{v}^{2} F(c \eta)+\sigma_{v}^{2} \tilde{F}(\eta)+\mu_{v} \sigma_{v} \tilde{f}(\eta)\right] \\
& =\sigma_{u v} \tilde{F}(\eta)+\mu_{u}\left[\mu_{v} F(c \eta)+\sigma_{v} \tilde{f}(\eta)\right] .
\end{aligned}
$$

This completes the proof.

The following lemma is used in proving the uniqueness of the solution to the equation in Proposition 1 for elliptically distributed random variables.

Lemma A.2. Let $f$ and $F$ be the density and cumulative distribution functions of a spherical random variable $\tilde{v}$ with finite variance. By truncating $\tilde{v}$ from above at the value of cu, we define a truncated random variable $x$ with density function $f(x) / F(c u)$ for $-\infty<x<c u$. The variance of $x$ is given by

$$
\operatorname{Var}[x]=\frac{c^{2}}{F(c u)}\left[\tilde{F}(u)-u \tilde{f}(u)-\frac{\tilde{f}(u)^{2}}{F(c u)}\right],
$$

where $c=\sigma_{\tilde{v}}$, and $\tilde{f}$ and $\tilde{F}$ are the density and cumulative distribution functions of another elliptical random variable $w$ that is associated with $\tilde{v}$ as defined in (A16).

Proof of Lemma A.2. Using the fact that

$$
\frac{\partial c \tilde{f}(x / c)}{\partial x}=-x f(x)
$$

we can easily obtain

$$
E[x]=\frac{1}{F(c u)} \int_{-\infty}^{c u} x f(x) \mathrm{d} q=-\left.\frac{c}{F(c u)} \tilde{f}\left(\frac{x}{c}\right)\right|_{-\infty} ^{c u}=-\frac{c \tilde{f}(u)}{F(c u)} .
$$


Then using integration by parts, we obtain the second moment of $x$ as

$$
\begin{aligned}
E\left[x^{2}\right] & =\frac{1}{F(c u)} \int_{-\infty}^{c u} x^{2} f(x) \mathrm{d} x \\
& =\frac{1}{F(c u)}\left[-\left.c x \tilde{f}\left(\frac{x}{c}\right)\right|_{-\infty} ^{c u}+\int_{-\infty}^{c u} c \tilde{f}\left(\frac{x}{c}\right) \mathrm{d} q\right] \\
& =\frac{1}{F(c u)}\left[-c^{2} u \tilde{f}(u)+c^{2} \tilde{F}(u)\right] .
\end{aligned}
$$

Then, the variance of $x$ is given by (A38). This completes the proof.

\section{Appendix B: Proofs of Main Results}

Proof of Proposition 1. Since $m_{y}=y-\tilde{\lambda}^{\prime} \tilde{r}$ follows an elliptical distribution with mean $\mu_{m}$ and variance $\sigma_{m}^{2}$, we can invoke Lemma A.1 to obtain

$$
E\left[\tilde{r} m_{y}^{+}\right]=\tilde{F}(\eta)\left(E[\tilde{r} y]-U \tilde{\lambda}+E[\tilde{r}] \sigma_{m}[g(\eta)-\eta]\right)
$$

where $\eta=\mu_{m} / \sigma_{m}$.

Using the first order condition $E\left[\tilde{r} m_{y}^{+}\right]=q$ and the expression of $U^{-1}$ in (13), we obtain $U^{-1} E[\tilde{r}]=q / R_{0}$ and

$$
\begin{aligned}
\tilde{\lambda} & =U^{-1} E[\tilde{r} y]+\sigma_{m}[g(\eta)-\eta] U^{-1} E[\tilde{r}]-\frac{1}{\tilde{F}(\eta)} U^{-1} q \\
& =\left[\begin{array}{c}
\frac{\mu_{y}-V_{r y}^{\prime} V_{r r}^{-1} \mu_{r}}{R_{0}} \\
V_{r r}^{-1} V_{r y}
\end{array}\right]+\left[\begin{array}{c}
\frac{\sigma_{m}[g(\eta)-\eta]}{R_{0}} \\
0_{N}
\end{array}\right]-\frac{1}{\tilde{F}(\eta)}\left[\begin{array}{c}
\frac{1+a}{R_{0}^{2}} \\
-\frac{V_{r r}^{-1} \mu_{r}}{R_{0}}
\end{array}\right] .
\end{aligned}
$$

Using (B2) and after simplification, we have

$$
m_{y}=y-\tilde{\lambda}^{\prime} \tilde{r}=z+\frac{1}{\tilde{F}(\eta)}\left[\frac{1}{R_{0}}-\frac{\mu_{r}^{\prime} V_{r r}^{-1}\left(r-\mu_{r}\right)}{R_{0}}\right]-\sigma_{m}[g(\eta)-\eta] \text {, }
$$

and the variance of $m_{y}$ is given by

$$
\sigma_{m}^{2}=\sigma_{z}^{2}+\frac{a}{R_{0}^{2} \tilde{F}(\eta)^{2}}
$$


With (B2) and (B4), we can see that once $\eta$ is identified, $\tilde{\lambda}$ will be uniquely determined and there is no need to solve $n$ nonlinear equations to obtain $\tilde{\lambda}$.

Now, using the fact that $m_{y}^{+}$prices the risk-free asset correctly, we have $E\left[m_{y}^{+}\right]=1 / R_{0}$. Then using (A23) to express $E\left[m_{y}^{+}\right]=\tilde{F}(\eta) \sigma_{m} g(\eta)$, we obtain

$$
g(\eta)=\frac{1}{\sigma_{m} R_{0} \tilde{F}(\eta)}
$$

Substituting $\sigma_{m}$ from (B4) into this expression, we can see that $\eta$ satisfies the following first order condition:

$$
g(u)=\left[a+\sigma_{z}^{2} R_{0}^{2} \tilde{F}(u)^{2}\right]^{-\frac{1}{2}} .
$$

For establishing the uniqueness of the solution to equation (B6), we need to show that (i) $g(u)>0$, (ii) $g(\infty)=\infty$, (iii) $g(-\infty)=0$, and (iv) $g^{\prime}(u)>0$. Condition (i) follows from (A23). Condition (ii) follows from the definition of $g$. For condition (iii), it is convenient to write

$$
g(u)=\frac{u F(c u)+\tilde{f}(u)}{\tilde{F}(u)}=\frac{g_{1}(u)}{g_{2}(u)} .
$$

Then,

$$
\begin{aligned}
g_{1}^{\prime}(u) & =F(c u), \\
g_{2}^{\prime}(u) & =\tilde{f}(u), \\
g_{1}^{\prime \prime}(u) & =c f(c u), \\
g_{2}^{\prime \prime}(u) & =-c u f(c u) .
\end{aligned}
$$

Using L'Hôpital's rule twice, we have

$$
\lim _{u \rightarrow-\infty} g(u)=\lim _{u \rightarrow-\infty} \frac{g_{1}^{\prime \prime}(u)}{g_{2}^{\prime \prime}(u)}=\lim _{u \rightarrow-\infty} \frac{c f(c u)}{-c u f(c u)}=\lim _{u \rightarrow-\infty}-\frac{1}{u}=0
$$

For (iv), taking the derivative of $g(u)$, we have

$$
g^{\prime}(u)=\frac{F(c u)}{\tilde{F}(u)^{2}}\left[\tilde{F}(u)-u \tilde{f}(u)-\frac{\tilde{f}(u)^{2}}{F(c u)}\right]>0,
$$


where the inequality is obtained by using $(\mathrm{A} 38)$ and the fact that $\operatorname{Var}[x]>0$. Since the left hand side of (B6) is positive and increasing in $\eta$, and the right hand side of (B6) is positive and decreasing in $\eta$, the solution to (B6) is unique.

Using (B5), we can express the vector of Lagrange multipliers as

$$
\tilde{\lambda}=\left[\begin{array}{c}
\frac{\mu_{y}-V_{r y}^{\prime} V_{r r}^{-1} \mu_{r}}{R_{0}}+\frac{1-\frac{\eta}{g(\eta)}}{R_{0}^{2} \tilde{F}(\eta)}-\frac{1+a}{R_{0}^{2} \tilde{F}(\eta)} \\
V_{r r}^{-1}\left(V_{r y}+\frac{\mu_{r}}{R_{0} \tilde{F}(\eta)}\right)
\end{array}\right]=\left[\begin{array}{c}
\frac{\mu_{y}-V_{r y}^{\prime} V_{r r}^{-1} \mu_{r}}{R_{0}}-\frac{a+\frac{\eta}{g(\eta)}}{R_{0}^{2} \tilde{F}(\eta)} \\
V_{r r}^{-1}\left(V_{r y}+\frac{\mu_{r}}{R_{0} \tilde{F}(\eta)}\right)
\end{array}\right] .
$$

The nonnegative admissible SDF that is closest to $y$ is $m_{y}^{+}$, where $m_{y}$ is defined in (B3). It follows that the squared constrained HJ-distance of $y$ is given by

$$
\delta_{+}^{2}=E\left[\left(y-m_{y}^{+}\right)^{2}\right]=E\left[y^{2}\right]-2 E\left[y m_{y}^{+}\right]+E\left[m_{y}^{+2}\right] .
$$

It is straightforward to show that

$$
E\left[y^{2}\right]=\sigma_{z}^{2}+\mu_{y}^{2}+V_{r y}^{\prime} V_{r r}^{-1} V_{r y}
$$

Using (A24) and the fact that $E\left[m_{y}^{+}\right]=1 / R_{0}$, we obtain

$$
\begin{aligned}
& E\left[y m_{y}^{+}\right]=\left(\sigma_{z}^{2}-\frac{V_{r y}^{\prime} V_{r r}^{-1} \mu_{r}}{R_{0} \tilde{F}(\eta)}\right) \tilde{F}(\eta)+\frac{\mu_{y}}{R_{0}}, \\
& E\left[m_{y}^{+2}\right]=E\left[m_{y} m_{y}^{+}\right]=\sigma_{m}^{2} \tilde{F}(\eta)+\frac{\mu_{m}}{R_{0}} .
\end{aligned}
$$

With these expressions, we obtain

$$
\begin{aligned}
\delta_{+}^{2}= & \sigma_{z}^{2}+\mu_{y}^{2}+V_{r y}^{\prime} V_{r r}^{-1} V_{r y}-2\left(\sigma_{z}^{2} \tilde{F}(\eta)-\frac{V_{r y}^{\prime} V_{r r}^{-1} \mu_{r}}{R_{0}}\right)-\frac{2 \mu_{y}}{R_{0}}+\sigma_{m}^{2} \tilde{F}(\eta)+\frac{\mu_{m}}{R_{0}} \\
= & \left(\mu_{y}-\frac{1}{R_{0}}\right)^{2}+\left(V_{r y}+\frac{\mu_{r}}{R_{0}}\right)^{\prime} V_{r r}^{-1}\left(V_{r y}+\frac{\mu_{r}}{R_{0}}\right) \\
& +\sigma_{z}^{2}-2 \sigma_{z}^{2} \tilde{F}(\eta)+\sigma_{m}^{2} \tilde{F}(\eta)+\frac{\mu_{m}}{R_{0}}-\frac{1+a}{R_{0}^{2}} \\
= & \delta^{2}+\sigma_{z}^{2}[1-\tilde{F}(\eta)]+\left(\sigma_{m}^{2}-\sigma_{z}^{2}\right) \tilde{F}(\eta)+\frac{\eta \sigma_{m}}{R_{0}}-\frac{1+a}{R_{0}^{2}} \\
= & \delta^{2}+\sigma_{z}^{2} \tilde{F}(-\eta)+\frac{a+\frac{\eta}{g(\eta)}}{R_{0}^{2} \tilde{F}(\eta)}-\frac{1+a}{R_{0}^{2}}
\end{aligned}
$$

where the last equality follows from (B4) and (B5). This completes the proof. 
Proof of Lemma 1. We first show that $\partial \Delta / \partial \eta=0$.

$$
\begin{aligned}
\frac{\partial \Delta}{\partial \eta} & =-\sigma_{z}^{2} \tilde{f}(-\eta)-\frac{a \tilde{f}(\eta)}{R_{0}^{2} \tilde{F}(\eta)^{2}}+\frac{R_{0}^{2} \tilde{F}(\eta) g(\eta)-\eta\left[R_{0}^{2} \tilde{F}(\eta) g^{\prime}(\eta)+R_{0}^{2} \tilde{f}(\eta) g(\eta)\right]}{R_{0}^{4} \tilde{F}(\eta)^{2} g(\eta)^{2}} \\
& =-\sigma_{z}^{2} \tilde{f}(\eta)-\frac{a \tilde{f}(\eta)}{R_{0}^{2} \tilde{F}(\eta)^{2}}+\frac{1}{R_{0}^{2} g(\eta) \tilde{F}(\eta)}-\frac{\eta g^{\prime}(\eta)}{R_{0}^{2} g(\eta)^{2} \tilde{F}(\eta)}-\frac{\eta \tilde{f}(\eta)}{R_{0}^{2} g(\eta) \tilde{F}(\eta)^{2}} \\
& =-\frac{\tilde{f}(\eta)}{R_{0}^{2} \tilde{F}(\eta)^{2}}\left[a+\sigma_{z}^{2} R_{0}^{2} \tilde{F}(\eta)^{2}\right]+\frac{1}{R_{0}^{2} g(\eta) \tilde{F}(\eta)}-\frac{\eta g^{\prime}(\eta)}{R_{0}^{2} g(\eta)^{2} \tilde{F}(\eta)}-\frac{\eta \tilde{f}(\eta)}{R_{0}^{2} g(\eta) \tilde{F}(\eta)^{2}} \\
& =-\frac{\tilde{f}(\eta)}{R_{0}^{2} \tilde{F}(\eta)^{2} g(\eta)^{2}}+\frac{g(\eta) \tilde{F}(\eta)-\eta F(c \eta)}{R_{0}^{2} \tilde{F}(\eta)^{2} g(\eta)^{2}} \\
& =-\frac{\tilde{f}(\eta)}{R_{0}^{2} \tilde{F}(\eta)^{2} g(\eta)^{2}}+\frac{\tilde{f}(\eta)}{R_{0}^{2} \tilde{F}(\eta)^{2} g(\eta)^{2}} \\
& =0 .
\end{aligned}
$$

The fourth equality follows because

$$
a+\sigma_{z}^{2} R_{0}^{2} \tilde{F}(\eta)^{2}=\frac{1}{g(\eta)^{2}}
$$

and

$$
g^{\prime}(\eta)=\frac{F(c \eta)-g(\eta) \tilde{f}(\eta)}{\tilde{F}(\eta)}
$$

which can be easily verified by using (A25) and (B13). This suggests that as far as the partial derivatives of $\Delta$ with respect to $\left(\sigma_{z}^{2}, a, R_{0}\right)$ are concerned, we can treat $\eta$ as a constant. Thus, it follows that

$$
\begin{aligned}
\frac{\partial \Delta}{\partial \sigma_{z}^{2}} & =\tilde{F}(-\eta)>0 \\
\frac{\partial \Delta}{\partial a} & =\frac{1}{R_{0}^{2} \tilde{F}(\eta)}-\frac{1}{R_{0}^{2}}=\frac{\tilde{F}(-\eta)}{R_{0}^{2} \tilde{F}(\eta)}>0 \\
\frac{\partial \Delta}{\partial R_{0}} & =\frac{2}{R_{0}^{3}}\left[1-\frac{a \tilde{F}(-\eta)}{\tilde{F}(\eta)}-\frac{\eta}{\tilde{F}(\eta) g(\eta)}\right] .
\end{aligned}
$$

This completes the proof.

Proof of Proposition 2. Let $\tilde{y}=\tilde{\gamma}^{\prime} \tilde{f}$ and $m_{\tilde{y}}=\tilde{y}-\tilde{\lambda}^{\prime} \tilde{r}$, where $\tilde{f}=\left[1, f^{\prime}\right]^{\prime}$. In addition, let $C=E\left[\tilde{f} \tilde{f}^{\prime}\right]$ and $D=E\left[\tilde{r} \tilde{f}^{\prime}\right]$. Differentiating

$$
\delta_{+}^{2}=E\left[\tilde{y}^{2}\right]-E\left[m_{\tilde{y}}^{+2}\right]-2 \tilde{\lambda}^{\prime} q
$$


with respect to $\tilde{\gamma}$ and $\tilde{\lambda}$, we obtain the following first order conditions:

$$
\begin{aligned}
C \tilde{\gamma}-E\left[\tilde{f} m_{\tilde{y}}^{+}\right] & =0_{K+1}, \\
E\left[\tilde{r} m_{\tilde{y}}^{+}\right] & =q .
\end{aligned}
$$

Let $\mu_{m}=E\left[m_{\tilde{y}}\right]$ and $\sigma_{m}^{2}=\operatorname{Var}\left[m_{\tilde{y}}\right]$. Invoking Lemma A.1, we have

$$
\begin{aligned}
E\left[\tilde{f} m_{\tilde{y}}^{+}\right] & =\tilde{F}(\eta)\left(C \tilde{\gamma}-D^{\prime} \tilde{\lambda}+\sigma_{m}[g(\eta)-\eta] E[\tilde{f}]\right), \\
E\left[\tilde{r} m_{\tilde{y}}^{+}\right] & =\tilde{F}(\eta)\left(D \tilde{\gamma}-U \tilde{\lambda}+\sigma_{m}[g(\eta)-\eta] E[\tilde{r}]\right),
\end{aligned}
$$

where $\eta=\mu_{m} / \sigma_{m}$. Putting the above expressions into the first order conditions, we obtain

$$
\left[\begin{array}{cc}
\tilde{F}(-\eta) C & \tilde{F}(\eta) D^{\prime} \\
\tilde{F}(\eta) D & -\tilde{F}(\eta) U
\end{array}\right]\left[\begin{array}{l}
\tilde{\gamma} \\
\tilde{\lambda}
\end{array}\right]=\left[\begin{array}{c}
\tilde{F}(\eta) \sigma_{m}[g(\eta)-\eta] E[\tilde{f}] \\
q-\tilde{F}(\eta) \sigma_{m}[g(\eta)-\eta] E[\tilde{r}]
\end{array}\right] .
$$

Let $H=\left[C+\tilde{F}(\eta)\left(D^{\prime} U^{-1} D-C\right)\right]^{-1}$. We can use the partitioned matrix inverse formula to write

$$
\left[\begin{array}{cc}
\tilde{F}(-\eta) C & \tilde{F}(\eta) D^{\prime} \\
\tilde{F}(\eta) D & -\tilde{F}(\eta) U
\end{array}\right]^{-1}=\left[\begin{array}{cc}
H & H D^{\prime} U^{-1} \\
U^{-1} D H & -\frac{1}{\tilde{F}(\eta)} U^{-1}+U^{-1} D H D^{\prime} U^{-1}
\end{array}\right]
$$

Using (13), we can easily verify that $U^{-1} E[\tilde{r}]=q / R_{0}$ and hence

$$
D^{\prime} U^{-1} E[\tilde{r}]=\frac{1}{R_{0}} D^{\prime} q=\frac{1}{R_{0}} E\left[R_{0} \tilde{f}\right]=E[\tilde{f}] .
$$

Using this identity, we can then show that

$$
\tilde{\gamma}=H D^{\prime} U^{-1} q
$$

From the partitioned matrix inverse formula and after some algebra, we can simplify the $H$ matrix as

$$
H=\left[\begin{array}{cc}
1+\mu_{f}^{\prime} P \mu_{f} & -\mu_{f}^{\prime} P \\
-P \mu_{f} & P
\end{array}\right]
$$

where $P=\left[V_{f f}-\tilde{F}(\eta) V_{f f \cdot r}\right]^{-1}$. Using this expression and (13), we can then rewrite (B34) as

$$
\tilde{\gamma}=\left[\begin{array}{cc}
1+\mu_{f}^{\prime} P \mu_{f} & -\mu_{f}^{\prime} P \\
-P \mu_{f} & P
\end{array}\right]\left[\begin{array}{cc}
R_{0} & \mu_{r}^{\prime} \\
\mu_{f} R_{0} & V_{r f}^{\prime}+\mu_{f} \mu_{r}^{\prime}
\end{array}\right]\left[\begin{array}{c}
\frac{(1+a)}{R_{0}^{2}} \\
-\frac{V_{r r}^{-1} \mu_{r}}{R_{0}}
\end{array}\right] .
$$


After some algebra, we can express $\tilde{\gamma}=\left[\tilde{\gamma}_{0}, \tilde{\gamma}_{1}^{\prime}\right]^{\prime}$ as

$$
\tilde{\gamma}_{1}=-\frac{1}{R_{0}} P V_{r f}^{\prime} V_{r r}^{-1} \mu_{r}, \quad \tilde{\gamma}_{0}=\frac{1}{R_{0}}-\tilde{\gamma}_{1}^{\prime} \mu_{f} .
$$

As a result, we can write $\tilde{y}=\frac{1}{R_{0}}+\tilde{\gamma}_{1}^{\prime}\left(f-\mu_{f}\right)$. Since the expression of $\tilde{\lambda}$ in $(27)$ also works for $\tilde{y}$, we can use $\mu_{\tilde{y}}=1 / R_{0}$, and $V_{r \tilde{y}}^{\prime} V_{r r}^{-1} \mu_{r}=\tilde{\gamma}_{1}^{\prime} V_{r f}^{\prime} V_{r r}^{-1} \mu_{r}=-\tilde{a}_{1} / R_{0}$ to obtain

$$
\tilde{\lambda}=\left[\begin{array}{c}
\frac{1+\tilde{a}_{1}}{R_{0}^{2}}-\frac{a+\frac{\eta}{g(\eta)}}{R_{0}^{2} \tilde{F}(\eta)} \\
V_{r r}^{-1}\left(V_{r f} \tilde{\gamma}_{1}+\frac{\mu_{r}}{R_{0} \tilde{F}(\eta)}\right)
\end{array}\right] .
$$

Note that we only need to solve for $\eta$ to obtain explicit expressions for $\tilde{\gamma}$ and $\tilde{\lambda}$. Defining $\epsilon=$ $\left(f-\mu_{f}\right)-V_{r f}^{\prime} V_{r r}^{-1}\left(r-\mu_{r}\right)$ as the unspanned components of the factors, we can write

$$
m_{\tilde{y}}=\tilde{\gamma}^{\prime} \tilde{f}-\tilde{\lambda}^{\prime} \tilde{r}=\tilde{\gamma}_{1}^{\prime} \epsilon-\frac{\mu_{r}^{\prime} V_{r r}^{-1}\left(r-\mu_{r}\right)}{R_{0} \tilde{F}(\eta)}+\frac{\eta}{R_{0} \tilde{F}(\eta) g(\eta)} .
$$

Using $E[\epsilon]=0_{K}$ and $\operatorname{Var}[\epsilon]=V_{f f \cdot r}$, we have

$$
\sigma_{m}^{2}=\operatorname{Var}\left[m_{\tilde{y}}\right]=\tilde{\gamma}_{1}^{\prime} V_{f f \cdot r} \tilde{\gamma}_{1}+\frac{a}{R_{0}^{2} \tilde{F}(\eta)^{2}}
$$

Since $m_{\tilde{y}}^{+}$prices the risk-free asset correctly, we have

$$
E\left[m_{\tilde{y}}^{+}\right]=\tilde{F}(\eta) \sigma_{m} g(\eta)=\frac{1}{R_{0}} .
$$

Then, plugging the expression of $\sigma_{m}^{2}$ from (B40) into (B41), we obtain

$$
g(\eta)=\left[a+\tilde{\gamma}_{1}^{\prime} V_{f f \cdot r} \tilde{\gamma}_{1} R_{0}^{2} \tilde{F}(\eta)^{2}\right]^{-\frac{1}{2}} .
$$

Using the expression for $\tilde{\gamma}_{1}$ in (B37) and rearranging terms, we can see that $\eta$ satisfies the following equation:

$$
g(u)=\left(a+\alpha^{\prime}\left[\frac{1}{\tilde{F}(u)} I_{K}-V_{f f}^{-\frac{1}{2}} V_{f f \cdot r} V_{f f}^{-\frac{1}{2}}\right]^{-2} \alpha\right)^{-\frac{1}{2}}
$$

where $\alpha=V_{f f}^{-1} V_{f f \cdot r}^{\frac{1}{2}} V_{r f}^{\prime} V_{r r}^{-1} \mu_{r}$. Since the left hand side is positive and increasing in $u$ and the right hand side is positive and decreasing in $u$ (because all the eigenvalues of $V_{f f}^{-\frac{1}{2}} V_{f f \cdot r} V_{f f}^{-\frac{1}{2}}$ are less than or equal to one), (B43) has a unique solution. Using (A24), it is straightforward to obtain

$$
\begin{aligned}
E\left[\tilde{y} m_{\tilde{y}}^{+}\right] & =\operatorname{Cov}\left[\tilde{y}, m_{\tilde{y}}\right] \tilde{F}(\eta)+\frac{1}{R_{0}^{2}}=\left[\tilde{\gamma}_{1}^{\prime} V_{f f \cdot r} \tilde{\gamma}_{1}+\frac{\tilde{a}_{1}}{R_{0}^{2} \tilde{F}(\eta)}\right] \tilde{F}(\eta)+\frac{1}{R_{0}^{2}}, \\
E\left[m_{\tilde{y}}^{+2}\right] & =E\left[m_{\tilde{y}} m_{\tilde{y}}^{+}\right]=\sigma_{m}^{2} \tilde{F}(\eta)+\frac{\mu_{m}}{R_{0}}
\end{aligned}
$$


The squared constrained HJ-distance is then given by

$$
\begin{aligned}
\delta_{+}^{2} & =E\left[\left(\tilde{y}-m_{\tilde{y}}^{+}\right)^{2}\right] \\
& =E\left[\tilde{y}^{2}\right]-2 E\left[\tilde{y} m_{\tilde{y}}^{+}\right]+E\left[m_{\tilde{y}}^{+2}\right] \\
& =\frac{1}{R_{0}^{2}}+\tilde{\gamma}_{1}^{\prime} V_{f f} \tilde{\gamma}_{1}-2\left[\tilde{\gamma}_{1}^{\prime} V_{f f \cdot r} \tilde{\gamma}_{1}+\frac{\tilde{a}_{1}}{R_{0}^{2} \tilde{F}(\eta)}\right] \tilde{F}(\eta)-\frac{2}{R_{0}^{2}}+\sigma_{m}^{2} \tilde{F}(\eta)+\frac{\mu_{m}}{R_{0}} \\
& =\tilde{\gamma}_{1}^{\prime} V_{f f} \tilde{\gamma}_{1}-2 \tilde{\gamma}_{1}^{\prime} V_{f f \cdot r} \tilde{\gamma}_{1} \tilde{F}(\eta)-\frac{1+2 \tilde{a}_{1}}{R_{0}^{2}}+\left[\tilde{\gamma}_{1}^{\prime} V_{f f \cdot r} \tilde{\gamma}_{1}+\frac{a}{R_{0}^{2} \tilde{F}(\eta)^{2}}\right] \tilde{F}(\eta)+\frac{\eta \sigma_{m}}{R_{0}} \\
& =\tilde{\gamma}_{1}^{\prime}\left[V_{f f}-\tilde{F}(\eta) V_{f f \cdot r}\right] \tilde{\gamma}_{1}-\frac{1+2 \tilde{a}_{1}}{R_{0}^{2}}+\frac{a}{R_{0}^{2} \tilde{F}(\eta)}+\frac{\eta}{R_{0}^{2} \tilde{F}(\eta) g(\eta)} \\
& =\frac{a+\frac{\eta}{g(\eta)}}{R_{0}^{2} \tilde{F}(\eta)}-\frac{1+\tilde{a}_{1}}{R_{0}^{2}},
\end{aligned}
$$

where the second last equality is obtained by using $\sigma_{m}=1 /\left[R_{0} \tilde{F}(\eta) g(\eta)\right]$ from (B41). Finally, we can easily see that the first element of $\tilde{\lambda}$ in (B38) is equal to $-\delta_{+}^{2}$. This completes the proof.

Proof of Lemma 2. The probability for $y$ to take on negative values is equal to

$$
P[y<0]=F\left(-\frac{c \mu_{y}}{\sigma_{y}}\right)=F\left(-\frac{c}{R_{0} \sigma_{y}}\right)=F\left(-\frac{c}{R_{0} \sqrt{\gamma_{1}^{\prime} V_{f f} \gamma_{1}}}\right),
$$

where $\mu_{y}$ and $\sigma_{y}$ are the mean and standard deviation of the SDF $y$, respectively. In contrast, the probability for $\tilde{y}$ to take on negative values is equal to

$$
P[\tilde{y}<0]=F\left(-\frac{c \mu_{\tilde{y}}}{\sigma_{\tilde{y}}}\right)=F\left(-\frac{c}{R_{0} \sigma_{\tilde{y}}}\right)=F\left(-\frac{c}{R_{0} \sqrt{\tilde{\gamma}_{1}^{\prime} V_{f f} \tilde{\gamma}_{1}}}\right),
$$

where $\mu_{\tilde{y}}$ and $\sigma_{\tilde{y}}$ are the mean and standard deviation of the $\operatorname{SDF} \tilde{y}$, respectively. The inequality holds because

$$
\begin{aligned}
R_{0}^{2} \tilde{\gamma}_{1}^{\prime} V_{f f} \tilde{\gamma}_{1} & =\mu_{r}^{\prime} V_{r r}^{-1} V_{r f}\left(V_{f f}-\tilde{F}(\eta) V_{f f \cdot r}\right)^{-1} V_{f f}\left(V_{f f}-\tilde{F}(\eta) V_{f f \cdot r}\right)^{-1} V_{r f}^{\prime} V_{r r}^{-1} \mu_{r} \\
& =\mu_{r}^{\prime} V_{r r}^{-1} V_{r f} V_{f f}^{\frac{1}{2}}\left(I_{K}-\tilde{F}(\eta) V_{f f}^{-\frac{1}{2}} V_{f f \cdot r} V_{f f}^{-\frac{1}{2}}\right)^{-2} V_{f f}^{\frac{1}{2}} V_{r f}^{\prime} V_{r r}^{-1} \mu_{r} \\
& \leq \mu_{r}^{\prime} V_{r r}^{-1} V_{r f} V_{f f}^{\frac{1}{2}}\left(I_{K}-V_{f f}^{-\frac{1}{2}} V_{f f \cdot r} V_{f f}^{-\frac{1}{2}}\right)^{-2} V_{f f}^{\frac{1}{2}} V_{r f}^{\prime} V_{r r}^{-1} \mu_{r} \\
& =\mu_{r}^{\prime} V_{r r}^{-1} V_{r f}\left(V_{r f}^{\prime} V_{r r}^{-1} V_{r f}\right)^{-1} V_{f f}\left(V_{r f}^{\prime} V_{r r}^{-1} V_{r f}\right)^{-1} V_{r f}^{\prime} V_{r r}^{-1} \mu_{r} \\
& =R_{0}^{2} \gamma_{1}^{\prime} V_{f f} \gamma_{1} .
\end{aligned}
$$


For (42), the first inequality, $\delta^{2} \leq \tilde{\delta}^{2}$, is obvious since $\gamma$ is chosen to minimize $\delta^{2}=e^{\prime} U^{-1} e$ but $\tilde{\gamma}$ is not. For the second inequality, $\tilde{\delta}^{2} \leq \delta_{+}^{2}$, note that for every $h \in L^{2}$ with $E\left[h^{2}\right]=1$, we have

$$
\min _{m \in \mathcal{M}^{+}}(E[\tilde{y} h]-E[m h])^{2} \leq \delta_{+}^{2}
$$

Consider a portfolio $\omega$ with unit second moment, i.e., $\omega^{\prime} U \omega=1$. When $\tilde{y}$ is the SDF, the squared pricing error of the portfolio is $\left(\omega^{\prime} \tilde{e}\right)^{2}$, and it is maximized when $\omega$ is chosen to be

$$
\omega^{*}=\frac{U^{-1} \tilde{e}}{\left(\tilde{e}^{\prime} U^{-1} \tilde{e}\right)^{\frac{1}{2}}} .
$$

Let $h=\omega^{* \prime} \tilde{r}$. Since $h$ is a linear combination of $\tilde{r}, E[m h]=\omega^{* \prime} E[m \tilde{r}]=\omega^{* \prime} q$ and the price of $h$ is the same for every $m \in \mathcal{M}^{+}$. It follows that

$$
\delta_{+}^{2} \geq \inf _{m \in \mathcal{M}^{+}}(E[\tilde{y} h]-E[m h])^{2}=(E[\tilde{y} h]-E[m h])^{2}=\left(\omega^{* \prime}(E[\tilde{y} \tilde{r}]-q)\right)^{2}=\left(\omega^{* \prime} \tilde{e}\right)^{2}=\tilde{\delta}^{2}
$$

This completes the proof. 


\section{References}

Bansal, Ravi, David A. Hsieh, and S. "Vish" Viswanathan, 1993, A new approach to international arbitrage pricing, Journal of Finance 48, 1719-1747.

Campbell, John, and John H. Cochrane, 2000, Explaining the poor performance of consumptionbased asset pricing models, Journal of Finance 55, 2863-2878.

Hamada, Mahmoud, and Emiliano A. Valdez, 2008, CAPM and option pricing with elliptically contoured distributions, Journal of Risk and Insurance 75, 387-409.

Hansen, Lars Peter, John C. Heaton, and Erzo G. J. Luttmer, 1995, Econometric evaluation of asset pricing models, Review of Financial Studies 8, 237-274.

Hansen, Lars Peter, and Ravi Jagannathan, 1991, Implications of security market data for models of dynamic economies, Journal of Political Economy 99, 225-262.

Hansen, Lars Peter, and Ravi Jagannathan, 1997, Assessing specification errors in stochastic discount factor models, Journal of Finance 52, 557-590.

Kan, Raymond, and Cesare Robotti, 2008, The exact distribution of the Hansen-Jagannathan bound, Working paper, University of Toronto.

Kan, Raymond, and Cesare Robotti, 2009, Model comparison using the Hansen-Jagannathan distance, Review of Financial Studies 22, 3449-3490.

Landsman, Zinovity M., and Emiliano A. Valdez, 2003, Tail conditional expectations for elliptical distributions, North American Actuarial Journal 7, 55-71.

Owen, Joel, and Ramon Rabinovitch, 1983, On the class of elliptical distributions and their applications to the theory of portfolio choice, Journal of Finance 38, 745-752.

Zhou, Guofu, 1993, Asset-pricing tests under alternative distributions, Journal of Finance 48, $1927-1942$. 


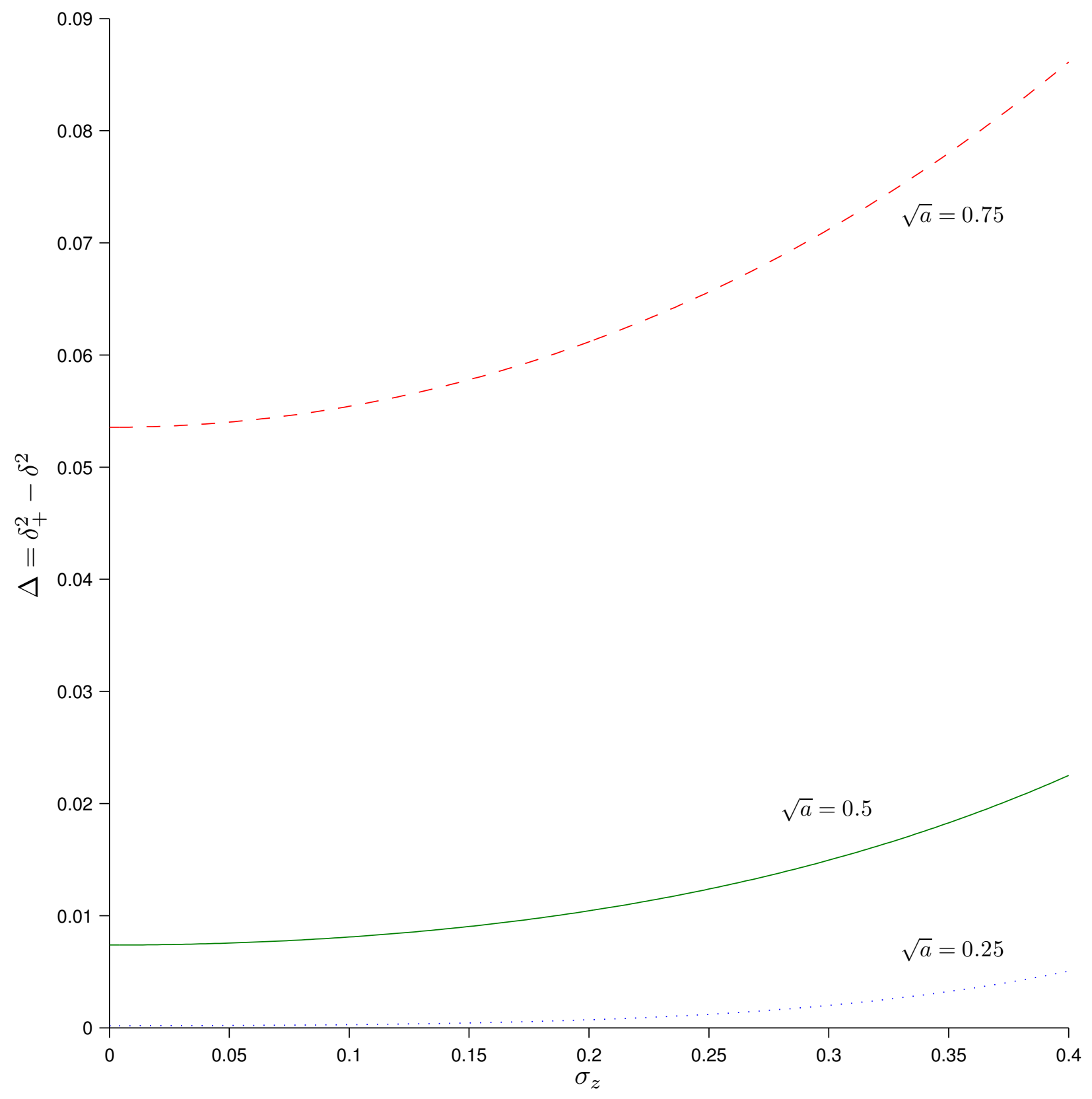

Figure 1. Difference between squared constrained and unconstrained HansenJagannathan distances. The figure plots the difference between squared constrained and unconstrained HJ-distances $(\Delta)$ as a function of the standard deviation of the unspanned component $\left(\sigma_{z}\right)$ of the candidate SDF. The gross risk-free rate is assumed to be 1.005. The dotted line represents the case in which the Sharpe ratio of the tangency portfolio $(\sqrt{a})$ is 0.25 . The solid line is for $\sqrt{a}=0.5$, and the dashed line is for $\sqrt{a}=0.75$. The SDF and the excess returns on the test assets are assumed to be multivariate $t$-distributed with six degrees of freedom. 


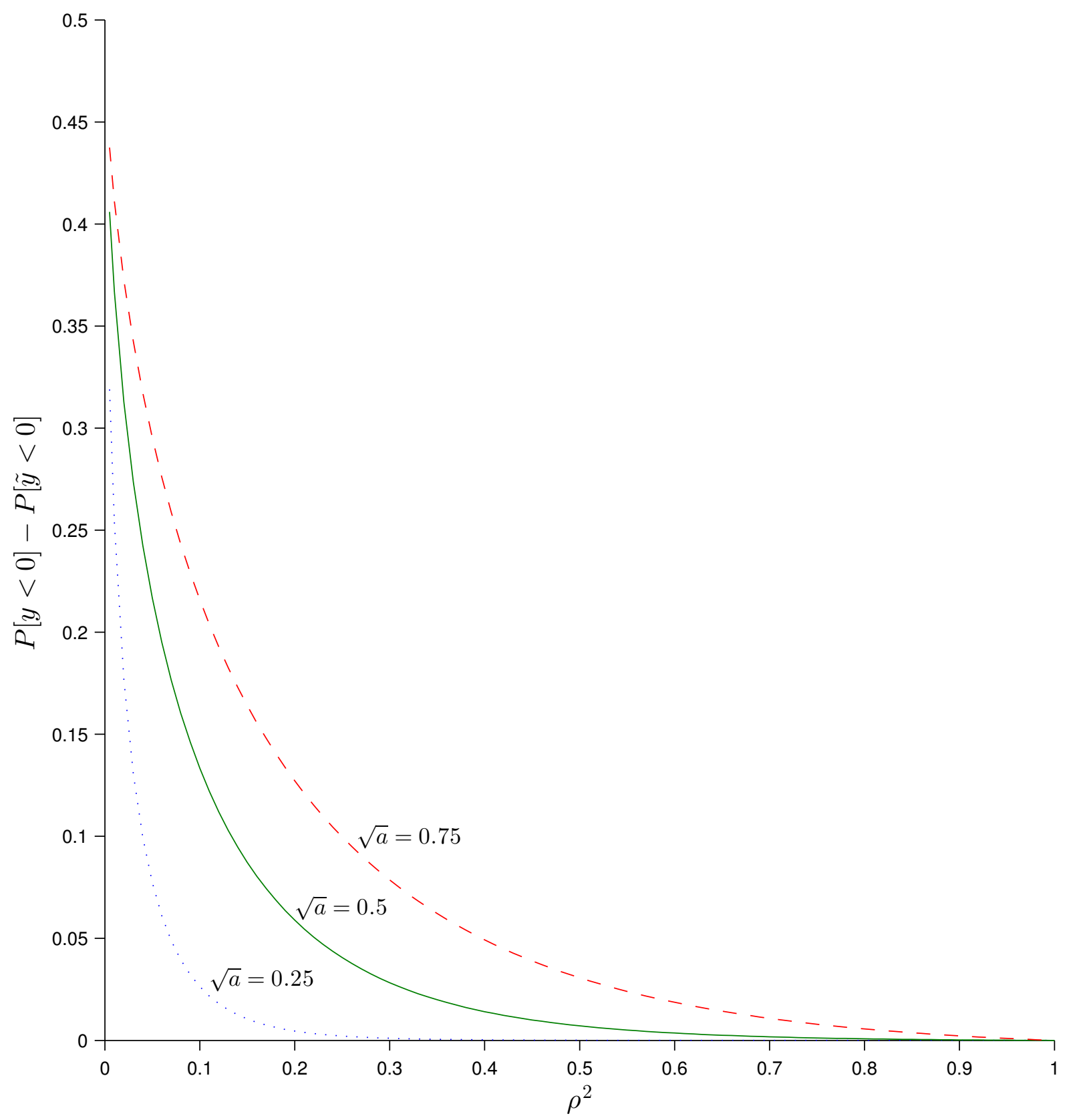

Figure 2. Difference in the probabilities of taking on negative values for two linear SDFs. The figure plots $P[y<0]-P[\tilde{y}<0]$ as a function of $\rho^{2}$ in a 1-factor setting, where $y$ and $\tilde{y}$ are the linear SDFs chosen to minimize the unconstrained and constrained HJ-distances, respectively. $\rho^{2}$ is the proportion of variability of the factor that is explained by the returns. The dotted line represents the case in which the Sharpe ratio of the tangency portfolio $(\sqrt{a})$ is 0.25 . The solid line is for $\sqrt{a}=0.5$, and the dashed line is for $\sqrt{a}=0.75$. In each case, we assume that the squared Sharpe ratio of the factor mimicking portfolio $\left(a_{1}\right)$ is half of the value of $a$. The factor and the excess returns on the test assets are assumed to be multivariate $t$-distributed with six degrees of freedom. 


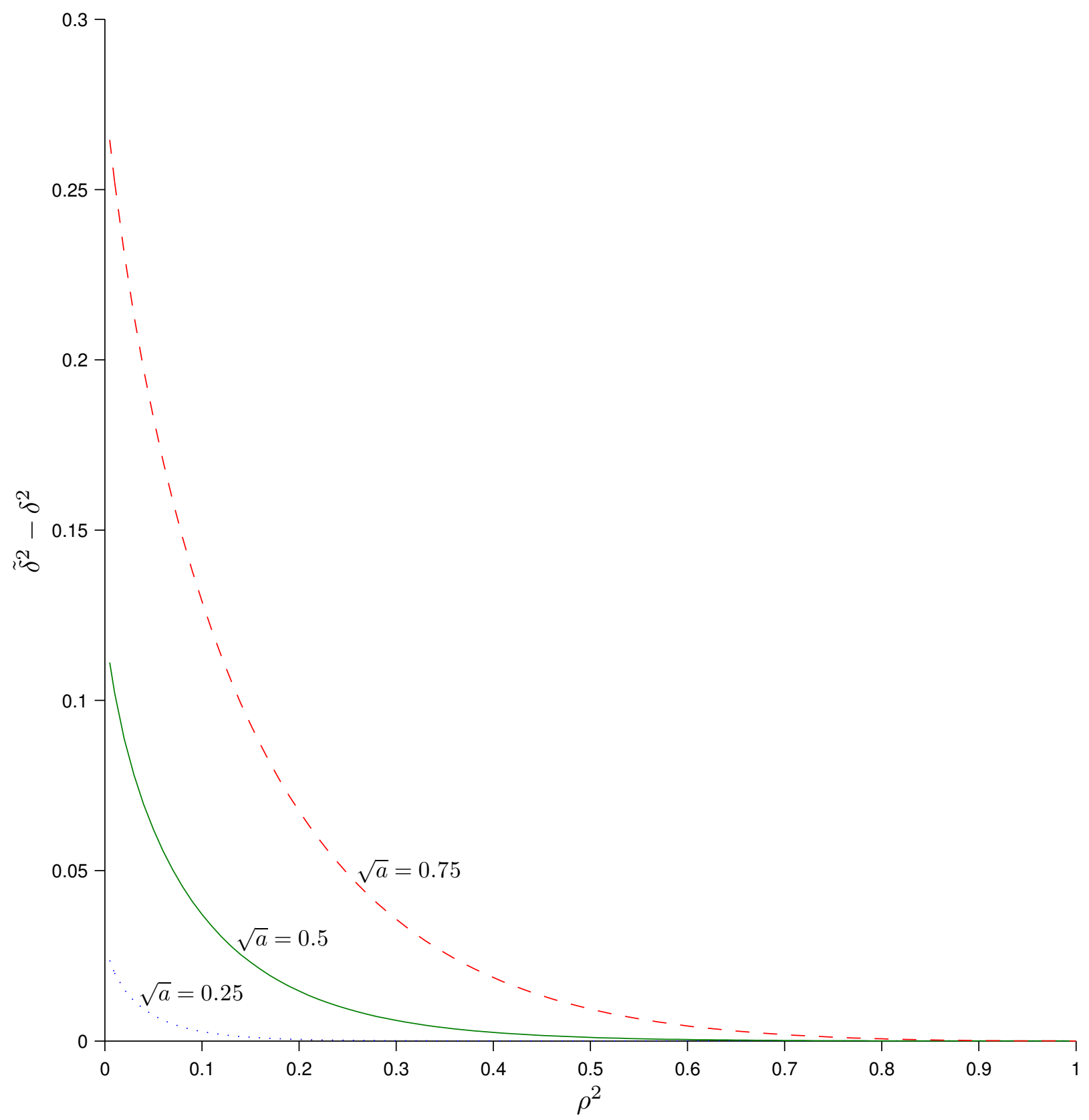

Figure 3. Difference in the aggregate measures of pricing errors of two linear SDFs. The figure plots $\tilde{\delta}^{2}-\delta^{2}$ as a function of $\rho^{2}$ in a one-factor setting, where $\delta^{2}$ and $\tilde{\delta}^{2}$ are the aggregate measures of pricing errors of the test assets when the linear SDF is chosen to minimize the unconstrained and constrained HJ-distances, respectively. $\rho^{2}$ is the proportion of variability of the factor that is explained by the returns. The dotted line represents the case in which the Sharpe ratio of the tangency portfolio $(\sqrt{a})$ is 0.25 . The solid line is for $\sqrt{a}=0.5$, and the dashed line is for $\sqrt{a}=0.75$. In each case, we assume that the squared Sharpe ratio of the factor mimicking portfolio $\left(a_{1}\right)$ is half of the value of $a$. The gross risk-free rate is assumed to be 1.005. The factor and the excess returns on the test assets are assumed to be multivariate $t$-distributed with six degrees of freedom. 